\title{
PSEUDOHOLOMORPHIC CURVES AND MULTIPLICITY OF HOMOCLINIC ORBITS
}

\author{
KAI CIELIEBAK AND ERIC SÉRÉ
}

1. Introduction. Let $M$ be a compact smooth manifold of dimension $n$. If we equip $M$ with a Riemannian metric, we get a 1 -form $\theta$ on the tangent bundle $T M \rightarrow^{\tau} M$ which we write in geodesic normal coordinates $\left(q_{i}, p_{i}\right)$ as

$$
\theta=\sum p_{i} d q_{i}
$$

and $\omega:=-d \theta$ is then a symplectic form on $T M$.

To $\omega$ and the Riemannian metric $\langle,>$ we associate an almost complex structure $J$ satisfying

$$
\omega(J \cdot, \cdot)=\langle\cdot, \cdot\rangle
$$

Let $H \in C^{\infty}(\mathbb{R} \times T M, \mathbb{R}), 1$-periodic in time, satisfy the following assumptions.

(H1) There exists an $x_{0}=\left(q_{0}, 0\right) \in T M$ such that

$$
\begin{gathered}
H\left(t, x_{0}\right)=0, \quad H^{\prime}\left(t, x_{0}\right)=0 \quad \text { for all } t, \\
H\left(t, q_{0}, p\right) \geqslant 0 \quad \text { for all } t, p, \\
H(t, q, 0)<0 \quad \text { for all } q \neq q_{0} .
\end{gathered}
$$

(H2) Let $M(t) \in \mathscr{N}_{2 n}(\mathrm{R})$ be the solution of the linearized system

$$
\begin{aligned}
\frac{d M}{d t} & =J\left(x_{0}\right) H^{\prime \prime}\left(t, x_{0}\right) M, \\
M(0) & =I .
\end{aligned}
$$

Then $M(1)$ has no eigenvalue of modulus 1 .

Cieliebak partially supported by Graduiertenkolleg Mathematische Physik and SFB 237, Bochum. 
(H3) There exists a vector field $\eta$ on $T M$ satisfying

$$
\begin{gathered}
d\left(i_{\eta} \omega\right)=\omega, \\
|\eta(x)| \leqslant c_{1}\left|x-x_{0}\right|, \\
\left\langle H^{\prime}(t, x), \eta(x)\right\rangle-H(t, x) \geqslant c_{2}\left|x-x_{0}\right|^{2} \quad \text { for some } c_{1}, c_{2}>0 .
\end{gathered}
$$

Here $|x-y|$ is a distance on $T M$ equivalent to the geodesic distance. Its precise definition is given at the beginning of Section 2 .

(H4) In geodesic normal coordinates around each $x=(q, p) \in T M$, we have

$$
\begin{gathered}
\left\langle\frac{\partial H}{\partial p}, p\right\rangle \geqslant c_{3}|p|^{2} \quad \text { for }|p| \geqslant p_{0}, c_{3}, p_{0}>0, \\
\left|\frac{\partial^{2} H}{\partial p^{2}}(t, x)\right|+\left|\frac{\partial^{2} H}{\partial p \partial q}(t, x)\right| \leqslant c_{4}, c_{4}>0 .
\end{gathered}
$$

The Hamiltonian system associated to $H$ is

$$
\dot{x}=J(x) H^{\prime}(t, x)=X_{H}(t, x) .
$$

Let

$$
\mathscr{C}=\left\{x \in C^{\infty}(\mathbb{R}, T M) \mid \dot{x}=X_{H}(t, x), \lim _{t \rightarrow \pm \infty} x(t)=x_{0}\right\}
$$

be the set of all solutions of the Hamiltonian system which are doubly asymptotic to $x_{0}$. This set contains the constant solution $x_{0}$. The elements of $\mathscr{C} \backslash\left\{x_{0}\right\}$ are called orbits homoclinic to $x_{0}$.

The goal of this paper is to prove some multiplicity results on these homoclinic orbits. Before stating the results, we have to explain how to distinguish the orbits. Actually, we will use two notions of multiplicity.

The first one is only meaningful for systems that are really nonautonomous. In that case, by periodicity of $H$ in time, there is a natural action of $\mathbf{Z}$ on $\mathscr{C}, n *$ $x(t)=x(t-n), n \in \mathbf{Z}, x \in \mathscr{C}$. We say that $x, y \in \mathscr{C}$ are geometrically distinct with respect to the time-1 map of the Hamiltonian system, if their classes in $\mathscr{C} / \mathbf{Z}$ are distinct. We will prove the following theorem.

TheOREM 1.1. Assume that (H1)-(H4) are satisfied. Then there are infinitely many orbits homoclinic to $x_{0}$, which are geometrically distinct with respect to the time-1 map of the Hamiltonian system.

When the Hamiltonian $H$ does not depend on time, the group of translations which acts on $\mathscr{C}$ is $\mathrm{R}$. We say that $x, y \in \mathscr{C}$ are geometrically distinct with respect 
to the flow of the Hamiltonian vector field $X_{H}(x)$ if their classes in $\mathscr{C} / \mathbb{R}$ are distinct. It is more difficult to obtain multiplicity in this sense. For instance, Theorem 1.1 only says that $\mathscr{C} / \mathbb{R}$ is nonempty.

Our second theorem implies the existence of several classes in $\mathscr{C} / \mathbb{R}$ for autonomous systems on manifolds having a large enough fundamental group $\pi_{1}$. It is a generalization of the works $[\mathrm{R}],[\mathrm{Fe}]$. In these papers, the Hamiltonian system is autonomous, the manifold $M$ is the torus $T^{n}$, and at least $2 n$ orbits are found. Their classes in $\pi_{1}\left(T^{n}\right)=Z^{n}$ are a family of generators of $Z^{n}$ considered as an additive structure.

THEOREM 1.2. Assume that (H1)-(H4) are satisfied. Then each element of $\pi_{1}(M)$ is a product of classes

$$
\left[\tau x^{m}\right] \cdot\left[\tau x^{m-1}\right] \cdots \cdot\left[\tau x^{1}\right]
$$

with $x^{i} \in \mathscr{C}$ for $1 \leqslant i \leqslant m$, and $\tau: T M \rightarrow M$ the canonical projection.

As a consequence, if $H$ does not depend on time, denoting by $g(M)$ the minimal number of generators of the multiplicative structure $\pi_{1}(M)$, there are at least $g(M)$ homoclinic orbits, geometrically distinct with respect to the flow of the Hamiltonian vector field $X_{H}(x)$.

Note that the assumptions (H1)-(H4) made in the two theorems above are satisfied, in particular, by Lagrangian systems, i.e., systems with Hamiltonian of the form $\left(|p|^{2} / 2\right)+V(t, q)$, where $V$ is smooth, $V\left(t, q_{0}\right)=0, V_{q}^{\prime}\left(t, q_{0}\right)=0, V_{q q}^{\prime \prime}\left(t, q_{0}\right)$ $<0$, and $V(t, q)<0$ for any $q \neq q_{0}$. In that case, we can take $\eta(q, p)=(0, p)$ in (H3).

Such systems have already been studied in [B], [R], [BGi], [GiR], where existence and multiplicity results were given. These works are based on the study of the Lagrangian functional $\int|\dot{q}|^{2}-V(q)$.

For systems satisfying (H1)-(H4) that are not of Lagrangian type, the only work we know is due to Felmer [Fe], in the case $M=T^{n}$.

To prove the theorems, we will study the " $L^{2}$-gradient lines" of the action functional $I(x):=-\int_{\mathbf{R}} x^{*}\left(i_{\eta} \omega\right)-\int_{\mathbf{R}} H(t, x(t)) d t$. This functional is well defined of class $C^{\infty}$ on the Hilbert manifold $H^{1,2}(\mathbb{R}, T M)=\left\{x \mid \int_{\mathbb{R}}\left(\left|x(t)-x_{0}\right|^{2}+|(d / d t) x(t)|^{2}\right)\right.$ is finite $\}$, and its nonconstant critical points are the homoclinic orbits. The $L^{2}$ gradient lines of $I$ are solutions of an elliptic problem. They are also called "pseudoholomorphic curves". They were introduced in symplectic geometry by Gromov [Gr], and play a central role in Floer's homology theory (see [F1]). Many of our arguments are similar to those of [Ci], where the study of pseudoholomorphic curves gives a multiplicity result on periodic orbits. We will also use some ideas of Hofer and Wysocki [HW], who proved the existence of a homoclinic orbit in $\mathbf{R}^{2 n}$ by a method of pseudoholomorphic curves.

When trying to obtain a multiplicity result for homoclinic orbits, the main difficulty one has to face is the lack of compactness of $I$ due to its invariance by translations in time. 
In the case of Theorem 1.1, it is possible to overcome this lack of compactness, because the group of translations is discrete. Theorem 1.1 will be proved by contradiction. More precisely, we will show that the following assumption is absurd:

$$
\begin{gathered}
\mathscr{C} / \mathbf{Z} \text { is finite. In other words, there is a finite set } \mathscr{P} \subset \mathscr{C} \backslash\left\{x_{0}\right\} \\
\text { such that }\{u(t-n) \mid u \in \mathscr{P}, n \in \mathbf{Z}\}=\mathscr{C} \backslash\left\{x_{0}\right\} .
\end{gathered}
$$

This kind of assumption was introduced in [CZES], and has already been used in several works (see, e.g., [S1], [S2], [CZR], [Li], [GiR]). It gives some additional compactness properties to functionals invariant by a discrete group of translations.

Unfortunately, in the case of autonomous systems, the group of translations is $\mathbb{R}$, and this strategy is inefficient. Theorem 1.2 will be proved by standard concentration-compactness arguments (see $[\mathrm{L}]$ ). Because of the lack of compactness, it seems very difficult to obtain stronger multiplicity results in the autonomous situation. For instance, we do not know what happens if $M$ is a sphere or a real projective space.

Remark. Instead of Theorem 1.1, one can prove a more precise result.

THEOREM 1.1'. Assume that $(H 1)-(H 4)$ are satisfied and that $\mathscr{C}$ is at most countable. Then there is a homoclinic orbit in each class of $\pi_{1}(M)$. If, moreover, $\pi_{1}(M)$ is finite, then there are infinitely many contractible orbits homoclinic to $x_{0}$, which are geometrically distinct with respect to the time-1 map of the Hamiltonian system.

This theorem corresponds to a weakening of assumption ( $\mathscr{H})$. Such a weakening has alread been detailed in [S2] for another type of Hamiltonian system, so we will not do it here. The modifications to make in the proof of Theorem 1.1 are minor and only concern Corollary 2.6, Lemma 2.7, the proof of Proposition 2.8, and the proof of Lemma 3.8.

Acknowledgement. This work is based on a suggestion by $\mathbf{H}$. Hofer. The authors thank Professor Hofer for his encouragements and for fruitful conversations. They also thank C. Viterbo for his interest in the subject and for his useful remarks.

2. Compactness of Palais-Smale sequences. We denote by $\mathbf{N}$ the set of natural numers including zero and by $H^{m, p}\left(\mathbb{R}^{a}, \mathbb{R}^{b}\right), m \in \mathbb{N}, a, b, p \geqslant 1$, the standard Sobolev spaces with Sobolev norms \|\|$_{m, p}$. In the case of functions with values in $T M$, we consider an isometric embedding $i$ of $M$ in $\mathbb{R}^{b}$ for some $b$, such that $i\left(q_{0}\right)=0$. Its differential $D i: T M \rightarrow \mathbb{R}^{2 b}$ is also an isometric embedding, and $D i\left(x_{0}\right)$ $=0$. Then we define, for $x, y \in T M$ and $f, g: \mathbb{R}^{a} \rightarrow T M$,

$$
\begin{gathered}
|x|=|D i(x)|_{\mathbf{R}^{2 b}}, \\
|x-y|=|D i(x)-D i(y)|_{\mathbf{R}^{2 b}},
\end{gathered}
$$




$$
\begin{gathered}
\|f\|_{m, p}=\|D i \circ f\|_{m, p}, \\
\|f-g\|_{m, p}=\|D i \circ f-D i \circ g\|_{m, p},
\end{gathered}
$$

and

$$
H^{m, p}\left(\mathbb{R}^{a}, T M\right)=\left\{f /\|f\|_{m, p} \text { is finite }\right\}
$$

Let $\mathscr{A}(t):=H^{\prime \prime}\left(t, x_{0}\right)$ and $J:=J\left(x_{0}\right)$ as in (H2).

Lemma 2.1. Assume that (H1)-(H4) are satisfied. Then, for any $m \in \mathbf{N}$, the operator

$$
\left(-J \frac{d}{d t}-\mathscr{A}(t)\right): H^{m+1,2}\left(\mathbb{R}, \mathbb{R}^{2 n}\right) \rightarrow H^{m, 2}\left(\mathbb{R}, \mathbb{R}^{2 n}\right)
$$

is an isomorphism.

Proof. Let $M(t)$ be the solution of

$$
\frac{d}{d t} M(t)=J \mathscr{A}(t) M(t), \quad M(0)=I
$$

Let $A_{0}$ be a matrix such that $e^{J A_{0}}=M(1)$.

Define

$$
P(t):=M(t) e^{-J A_{0} t}
$$

If we denote by $P(t)^{*}$ its conjugate matrix, it follows from straightforward computations that, for any $z \in C^{1}\left(\mathbf{R}, \mathbf{R}^{2 n}\right)$,

$$
P(t) *\left[J \frac{d}{d t}+\mathscr{A}(t)\right] P(t) z(t)=\left(J \frac{d}{d t}+A_{0}\right) z(t) .
$$

Since $P(t)$ is invertible and $P(1)=P(0)$, multiplication by $P(t)$ or $P(t)^{*}$ gives isomorphisms of $H^{m, 2}\left(\mathbb{R}, \mathbb{R}^{2 n}\right)$ into itself. Thus it suffices to show that

$$
\left(-J \frac{d}{d t}-A_{0}\right): H^{m+1,2}\left(\mathbf{R}, \mathbf{R}^{2 m}\right) \rightarrow H^{m, 2}\left(\mathbf{R}, \mathbf{R}^{2 m}\right)
$$

is an isomorphism.

But $A_{0}$ is time independent, and from $\left(\mathrm{H}_{2}\right)$ we deduce that $J A_{0}$ has no purely imaginary eigenvalues. The result now follows by Fourier transformation. 
488

CIELIEBAK AND SERE

For $x \in H^{1,2}(\mathbb{R}, T M)$, we define the action

$$
I(x):=-\int_{\mathbf{R}} x^{*}\left(i_{\eta} \omega\right)-\int_{\mathbf{R}} H(t, x(t)) d t .
$$

Using the $L^{2}$ product $\langle x, y\rangle_{L^{2}}:=\int_{\mathbb{R}}\langle x(t), y(t)\rangle d t$, the $L^{2}$-gradient $I^{\prime}(x) \in L^{2}(\mathbb{R}$, $\left.x^{*} T T M\right)$ is defined implicitly by the formula

$$
d I(x) \xi=\left\langle I^{\prime}(x), \xi\right\rangle_{L^{2}} \text { for all } \xi \in H^{1,2}\left(\mathbb{R}, x^{*} T T M\right)
$$

Here

$$
d I(x) \xi:=\left.\frac{d}{d s}\right|_{s=0} I(\exp s \xi) .
$$

With the help of the almost complex structure $J$, we can write $I^{\prime}(x)$ explicitly as

$$
I^{\prime}(x)=-J(x) \dot{x}-H^{\prime}(t, x) .
$$

The key to the Palais-Smale property is given by the following easy lemma.

LEMMA 2.2. Assume that (H1)-(H4) are satisfied. Then

$$
\|x\|_{1,2}^{2} \leqslant K\left(\left\|I^{\prime}(x)\right\|_{L^{2}}^{2}+I(x)\right) \quad \text { for all } x \in H^{1,2}(\mathbb{R}, T M) .
$$

Proof.

$$
\begin{aligned}
I(x)+c_{1}\left\|x-x_{0}\right\|_{L_{2}}\left\|I^{\prime}(x)\right\|_{L^{2}} \geqslant & I(x)+\left\|I^{\prime}(x)\right\|_{L^{2}}\|\eta(x)\|_{L^{2}} \\
\geqslant & I(x)-\left\langle I^{\prime}(x), \eta(x)\right\rangle_{L_{2}} \\
= & -\int x^{*}\left(i_{\eta} \omega\right)-\int H(t, x) d t \\
& -\int \omega\left(\dot{x}-J(x) H^{\prime}(t, x), \eta(x)\right) d t \\
= & -\int \omega(\eta, \dot{x})-\int H(t, x) d t-\int \omega(\dot{x}, \eta) \\
& +\int\left\langle H^{\prime}(t, x), \eta\right\rangle d t \\
\geqslant & c_{2}\left\|x-x_{0}\right\|_{L^{2}}^{2} .
\end{aligned}
$$


So

$$
\left(\left\|x-x_{0}\right\|_{L^{2}}-\frac{c_{1}}{2 c_{2}}\left\|I^{\prime}(x)\right\|_{L^{2}}\right)^{2} \leqslant \frac{1}{c_{2}} I(x)+\left(\frac{c_{1}}{2 c_{2}}\right)^{2}\left\|I^{\prime}(x)\right\|_{L^{2}}^{2},
$$

and thus

$$
\left\|x-x_{0}\right\|_{L^{2}}^{2} \leqslant K_{1}\left(\left\|I^{\prime}(x)\right\|_{L^{2}}^{2}+I(x)\right)
$$

Moreover, we have $d x / d t=J(x)^{-1}\left[-H^{\prime}(t, x)-I^{\prime}(x)\right]$. So

$$
\left\|\frac{d x}{d t}\right\|_{L^{2}}^{2} \leqslant K_{2}\left(\left\|x-x_{0}\right\|_{L^{2}}^{2}+\left\|I^{\prime}(x)\right\|_{L^{2}}^{2}\right)
$$

Hence the result.

We now give a bound from below on the norm of homoclinic orbits.

LemMa 2.3. Assume that $(H 1)-(H 4)$ are satisfied. Then there exists a $\rho>0$ such that

$$
I(x) \geqslant \rho \text { and }\|x\|_{L^{2}} \geqslant \rho \text { for all } x \in \mathscr{C} \backslash\left\{x_{0}\right\}
$$

Proof. From assumption (H2), M(1) is hyperbolic, so the time-1-map of the Hamiltonian system between time 0 and time 1 is $C^{0}$-close to the map $y \mapsto M(1) y$, in a neighborhood $\mathscr{V}$ of $x_{0}$. Moreover, there is no orbit $\left(M(1)^{n} y\right)_{n \in z}$ that remains in a bounded neighborhood of 0 for all values of $n$, except the null orbit. So there is no homoclinic orbit to $x_{0}$ that remains in $\mathscr{V}$. This gives the desired estimate on $\|x\|_{L^{2}}$. Then the estimate on $I(x)$ follows from Lemma 2.2 .

LEMMA 2.4. Assume that (H1)-(H4) are satisfied. Let $\left(x_{n}\right) \in H^{1,2}$ be a sequence such that $I\left(x_{n}\right) \rightarrow c \neq 0$ and $\left\|I^{\prime}\left(x_{n}\right)\right\|_{L^{2}} \rightarrow 0$. Then, after extraction, there are a sequence of integer shifts $\left\{\tau_{n}, n \in \mathbb{N}\right\}$ and a homoclinic orbit $x(t)$ such that $x_{n}\left(t+\tau_{n}\right) \rightarrow x(t)$ in the $H_{\text {loc }}^{1,2}$-topology.

Proof. First step. There is a sequence of shifts $\left(\tau_{n}\right)_{n \geqslant 0}$ such that $\left|x_{n}\left(\tau_{n}\right)-x_{0}\right|$ $\geqslant \rho$ (for some fixed $\rho>0$ ).

Otherwise we would have $\left\|x_{n}-x_{0}\right\|_{L^{\infty}} \rightarrow 0$ (after extraction). Then, for $n$ large enough, $x_{n}$ would remain in a neighborhood of $x_{0}$, and, in local coordinates around $x_{0}$, we would have (identifying $x_{0}$ with 0 )

$$
-J\left(x_{n}\right) \frac{d x_{n}}{d t}-\mathscr{A}(t) x_{n}=R_{x}^{\prime}\left(t, x_{n}\right)+I^{\prime}\left(x_{n}\right),
$$


where

$$
R(t, x)=H(t, x)-\frac{1}{2}\langle x, \mathscr{A}(t) x\rangle
$$

satisfies

$$
\left|R_{x}^{\prime}(t, x)\right| \leqslant C|x|^{2}
$$

So we could write

$$
\left(-J\left(x_{0}\right) \frac{d}{d t}-\mathscr{A}\right) x_{n}=R_{x}^{\prime}\left(t, x_{n}\right)+\left(J\left(x_{n}\right)-J\left(x_{0}\right)\right) \dot{x}_{n}+I^{\prime}\left(x_{n}\right) .
$$

We know that

$$
\begin{aligned}
&\left\|I^{\prime}\left(x_{n}\right)\right\|_{L^{2}} \rightarrow 0, \\
&\left\|R_{x}^{\prime}\left(t, x_{n}\right)\right\|_{L^{2}} \leqslant C\left\|x_{n}\right\|_{\infty}\left\|x_{n}\right\|_{L^{2}} \rightarrow 0, \\
&\left\|\left(J\left(x_{n}\right)-J\left(x_{0}\right)\right) \dot{x}_{n}\right\|_{L^{2}} \leqslant\left\|J\left(x_{n}\right)-J\left(x_{0}\right)\right\|_{L^{\infty}}\left\|\dot{x}_{n}\right\|_{L^{2}} \rightarrow 0 .
\end{aligned}
$$

So, from Lemma 2.1, $\left\|x_{n}\right\|_{H^{1,2}} \rightarrow 0$, and hence $I\left(x_{n}\right) \rightarrow 0$, which gives a contradiction.

Second step. Taking the sequence of shifts of the first step, we know from Lemma 2.2 that $\tilde{x}_{n}=x_{n}\left(t+\tau_{n}\right)$ is compact in the $C_{\text {loc }}^{0}$-topology. Let $x$ be its limit after extraction. We have $\left|x(0)-x_{0}\right| \geqslant \rho$. Each $\tilde{x}_{n}$ satisfies

$$
\int_{0}^{t} J\left(x_{n}\right) H^{\prime}\left(t, x_{n}\right)+\int_{0}^{t} J\left(x_{n}\right) I^{\prime}\left(x_{n}\right)=x_{n}(t)-x_{n}(0) .
$$

Then $\int_{0}^{t} J(x) H^{\prime}(t, x)=x(t)-x(0)$ for any $t \in \mathbb{R}$. So $x$ is solution of the Hamiltonian system and is limit of $\tilde{X}_{n}$ on the $H_{\text {loc }}^{1,2}$ topology.

Moreover,

$$
\left(\int\left|\tilde{x}_{n}-x_{0}\right|^{2}+\left|\dot{\tilde{x}}_{n}\right|^{2}\right)^{1 / 2} \leqslant C^{\prime}\left(\left\|I^{\prime}\left(\tilde{x}_{n}\right)\right\|_{L^{2}}+\left|I\left(\tilde{x}_{n}\right)\right|^{1 / 2}\right) .
$$

So $\left(\int\left|x-x_{0}\right|^{2}+|\dot{x}|^{2}\right)^{1 / 2} \leqslant C^{\prime \prime}$, and $x$ is a homoclinic orbit.

We are now in a position to state our compactness results. From now on, we suppose that $(\mathscr{H})$ is satisfied (see the end of Section 1 ).

Let $\varphi: \mathscr{Q} \subset \mathbb{R}^{2 n} \rightarrow B\left(x_{0}, \varepsilon_{0}\right)$ be a local map with $\varphi(0)=x_{0}$, and let $f: \mathbb{R} \rightarrow$ $[0,1]$ be a smooth function such that $f((-\infty, 0])=1, f([1,+\infty))=0$. Given $x \in$ $H^{1,2}(\mathbb{R}, T M)$, there is $\mathscr{R}(x)$ such that $\left|x(t)-x_{0}\right|<\varepsilon_{0}$ for any $t \in \mathbb{R} \backslash[-\mathscr{R}(x), \mathscr{R}(x)]$. 
So, for any $R \geqslant \mathscr{R}(x)$, we can define $x_{R}(t)=x(t)$ for $|t| \leqslant R$, and $x_{R}(t)=\varphi(f(|t|-$ R) $\left.\varphi^{-1}(x(t))\right)$ for $|t| \geqslant R$.

Now, given $m$ homoclinic orbits $x^{1}, \ldots, x^{m}$, and $m$ integers $p^{1}, \ldots, p^{m}$, such that

$$
(\forall i)\left(p^{i+1}-p^{i}\right) \geqslant 2 \max \left(\mathscr{R}\left(x^{i}\right), \mathscr{R}\left(x^{i+1}\right)\right)+2,
$$

we define the formal sum of bumps

$$
\bar{p} * \bar{x}=\sum_{i=1}^{m} p^{i} * x^{i}
$$

as the function satisfying

$$
\bar{p} * \bar{x}=x_{R_{i}}^{i}\left(t-p^{i}\right) \text { for } t \in\left[\frac{p_{i-1}+p_{i}}{2}, \frac{p_{i}+p_{i+1}}{2}\right],
$$

with $p_{0}=-\infty, p_{m+1}=+\infty$, and

$$
R_{i}=\min \left(\frac{p_{i}-p_{i-1}}{2}, \frac{p_{i+1}-p_{i}}{2}\right)-1
$$

As a consequence of the above lemmas, we have the following result.

Proposition 2.5. Assume that $(H 1)-(H 4)$ are satisfied. Let $\left(x_{n}\right)$ be a sequence such that

$$
\begin{aligned}
& I\left(x_{n}\right) \leqslant K, \\
& I^{\prime}\left(x_{n}\right) \underset{L^{2}}{\overrightarrow{2}} 0 .
\end{aligned}
$$

Then there exist an integer $m$, an $m$-tuple $\bar{x}=\left(x^{1}, \ldots, x^{m}\right) \in \mathscr{C}^{m}$, and a sequence $\overline{p_{n}}=\left(p_{n}^{1}, \ldots, p_{n}^{m}\right) \in \mathbf{Z}^{m}$ satisfying

$$
\left(p_{n}^{i+1}-p_{n}^{i}\right) \underset{n \rightarrow \infty}{\longrightarrow}+\infty \quad(\forall i),
$$

and such that, for some subsequence $x_{\varphi(n)}$ of $\left(x_{n}\right)$,

$$
\left\|x_{\varphi(n)}-\overline{p_{n}} * \bar{x}\right\|_{H^{1,2}(\mathbf{R}, T M)} \rightarrow 0 \text {. }
$$

Proof. This type of result is standard for problems exhibiting a lack of compactness due to an invariance by translations. See, for instance, [L], [St], [CZES], [GiR]. We will not give the complete proof because it would not contain any new ideas. The interested reader is referred to these papers for more details.

Proposition 2.5 has an immediate corollary. 
COROLlaRY 2.6. Assume that (H1)-(H4) and (H) are satisfied. Let $\mathscr{P} \subset \mathscr{C}$ be a finite set such that $\mathscr{C} \backslash\left\{x_{0}\right\}=\mathbf{Z} * \mathscr{P}$.

Given $K>0, \Delta>0$, and $\alpha>0$, there is $\varepsilon(K, \Delta, \alpha)>0$ such that, if $|I(x)| \leqslant K$ and $\left\|I^{\prime}(x)\right\|_{L^{2}} \leqslant \varepsilon$, then

$$
\|x-\bar{p} * \bar{x}\|_{H^{1,2}(\mathbf{R}, T M)} \leqslant \alpha
$$

for some $m \in \mathbb{N}, \bar{x}=\left(x^{1}, \ldots, x^{m}\right) \in \mathscr{P}^{m}$, and $\bar{p}=\left(p^{1}, \ldots, p^{m}\right) \in Z^{m}$ satisfying

$$
\left(p^{i+1}-p^{i}\right) \geqslant \Delta \quad(\forall i)
$$

Moreover, the integer $m$ (possibly 0 ) is bounded from above by a constant $M(K)$.

Now, for $y \in H^{1,2}(\mathbb{R}, T M), \rho>0$, let

$$
B_{L^{2}}(y, \rho):=\left\{x \in H^{1,2}(\mathbb{R}, T M) /\|x-y\|_{L^{2}}<\rho\right\},
$$

where $\|x-y\|_{L^{2}}$ was defined at the beginning of this section.

We have the following "annulus lemma".

LemMa 2.7. Assume that (H1)-(H4) and $(\mathscr{H})$ are satisfied. Then there exists an $r_{0}>0$ such that

$$
\|x-y\|_{L^{2}} \geqslant 3 r_{0} \text { for all } x \neq y \in \mathscr{C}
$$

Moreover, for any $\rho \in\left(0, r_{0}\right)$,

$$
\inf \left\{\left\|I^{\prime}(x)\right\|_{L^{2}} \mid x \in \bigcup_{y \in \varangle} B_{L^{2}}\left(y, r_{0}\right) \backslash B_{L^{2}}(y, \rho)\right\}>0
$$

Similarly, for any $\bar{y}=\left(y^{1}, \ldots, y^{m}\right) \in \mathscr{C}^{m}, m>0$,

$$
\liminf _{\bar{k} \rightarrow \Omega}\left[\inf \left\{\left\{\left\|I^{\prime}(x)\right\|_{L^{2}} \mid x \in B_{L^{2}}\left(\bar{k} * \bar{y}, r_{0}\right) \backslash B_{L^{2}}(\bar{k} * \bar{y}, \rho)\right\}\right]>0\right.
$$

Here $\bar{k} \rightarrow \Omega$ means $\left(k^{i+1}-k^{i}\right) \rightarrow+\infty$ for all $i \in[1, \ldots, m]$.

Proof. The proof is analogous to that of [S1], Lemma 8.

Corollary 2.6 and Lemma 2.7 give us the following result.

Proposirion 2.8. Assume that $(H 1-4)$ and $(\mathscr{H})$ are satisfied. Let $\left(x_{n}\right) \subset$ $H^{1.2}(\mathbb{R}, T M)$ with $I\left(x_{n}\right) \rightarrow c,\left\|I^{\prime}\left(x_{n}\right)\right\|_{L^{2}} \rightarrow 0$, and $\left\|x_{n}-x_{n+1}\right\|_{L^{2}} \rightarrow 0$. Then $x_{n}$ converges in $H^{1.2}$ to an $x \in \mathscr{B}$ with $I(x)=c$.

Proof. The proof is analogous to that of [S1], Lemma 4. 
3. Compactness of flow lines. In this section we establish compactness properties for two types of flow lines of the $L^{2}$-gradient $I^{\prime}$. On the one hand, we shall study flow lines of finite length connecting two subspaces of $H^{1,2}(\mathbb{R}, T M)$, namely the curves in the zero section and the curves lying over some fixed base curve $\bar{q} \in H^{1,2}(\mathbb{R}, M) \cap C^{\infty}$. More precisely, we fix a $T>0$ and define

$$
\Omega_{T}^{\infty}:=\left\{\bar{q} \in C^{\infty}(\mathbb{R}, M) \mid \bar{q}(t) \equiv q_{0} \text { for }|t| \geqslant T\right\},
$$

which we equip with the $C^{\infty}$ topology. The introduction of this space is inspired by [Fe]. For a fixed $\vec{q} \in \Omega_{T}^{\infty}$ and $R>0$, we set $Z_{R}=[0, R] \times \mathbf{R}$ and

$$
\mathscr{E}_{\bar{q}, R}:=\left\{u \in H^{2,2}\left(Z_{R}, T M\right) \mid u(0, t) \in M, u(R, t) \in T_{\bar{q}(t)} M \text { for all } t \in \mathbb{R}\right\},
$$

where $M \subset T M$ denotes the zero section. Let $\mathscr{E}_{\bar{q}, R}$ be equipped with the $H^{2,2}$ topology. For $u: Z_{R} \rightarrow T M$ we define

$$
\bar{\partial} u:=\partial_{s} u+J(u) \partial_{t} u,
$$

where $(s, t)$ are the canonical coordinates on $Z_{R}$.

We are interested in the finite flow lines

$$
X_{\bar{q}, R}:=\left\{u \in \mathscr{E}_{\bar{q}, R} \mid \bar{\partial} u+H^{\prime}(t, u)=0\right\} .
$$

Note that $\bar{\partial} u+H^{\prime}(u)=\partial_{s} u-I^{\prime}(u)$, so the elements of $X_{\bar{q}, R}$ are indeed "flow lines" for $I^{\prime}$.

Simultaneously we will study infinite flow lines connecting critical points of I. In order to get compactness results we must be careful about the choice of topology.

To a function $u: \mathbb{R}^{2} \rightarrow T M$, we associate, when it is defined, the expression

$$
\|u\|_{\text {semiloc }}=\left(\sum_{n \geqslant 1} \frac{1}{2^{n}}\|u\|_{H^{m, p}([-n, n] \times R, T M)}^{p}\right)^{1 / p}
$$

for $m \geqslant 0, p \geqslant 1$. Let

$$
\mathscr{E}=\left\{u: \mathbb{R}^{2} \rightarrow T M \mid\|u\|_{\text {semiloc }}^{2,2} \text { is finite }\right\},
$$

and

$$
X=\left\{u \in \mathscr{E} \mid \bar{\partial} u+H^{\prime}(t, u)=0 \text { and } \int_{\mathbb{R}^{2}}\left|\partial_{s} u\right|^{2} d s d t<\infty\right\}
$$

$\mathscr{E}$ and $X$ are endowed with the topology associated to the distance $d_{\ell}(u, v)=$ $\|u-v\|_{\substack{m, p \\ \text { semiloc }}}$. Note that $\mathscr{E}$ is a Hilbert manifold. 
LEMMA 3.1. Assume that (H1)-(H4) are satisfied. Let $u \in H^{2,2}([a, b] \times \mathbf{R}, T M)$ be such that

$$
\bar{\partial} u+H^{\prime}(t, u)=0 \text { and } 0 \leqslant I(u(a)) \leqslant I(u(b)) \leqslant A .
$$

Then, for some $C>0$ independent of $u, a, b$,

$$
\|u\|_{H^{1,2}([a, b] \times \mathbf{R}, T M)}^{2} \leqslant C(1+b-a) A .
$$

Proof. Note that $\left\|\partial_{s} u\right\|_{L^{2}(d s d t)}^{2}=I(u(b))-I(u(a)) \leqslant A$. From Lemma 2.2, for any $s \in[a, b]$,

$$
\begin{aligned}
\|u(s)\|_{H^{1.2}(d t)}^{2} & \leqslant K\left(\left\|\partial_{s} u(s, \cdot)\right\|_{L^{2}(d t)}^{2}+I(u(s))\right) \\
& \leqslant K\left(\left\|\partial_{s} u(s, \cdot)\right\|_{L^{2}(d t)}^{2}+A\right)
\end{aligned}
$$

and by integration over $s$

$$
\begin{aligned}
\|u\|_{H^{1,2}([a, b] \times R, T M)}^{2} & =\left\|\partial_{s} u\right\|_{L^{2}(d s d)}^{2}+\int_{a}^{b} d s\|u(s)\|_{H^{1,2}(d t)}^{2} \\
& \leqslant A+K A+(b-a) K A \\
& \leqslant C(1+b-a) A .
\end{aligned}
$$

Let us come back to infinite flow lines. The definition of $X$ implies that $\lim _{s \rightarrow \pm \infty} I(u(s))$ are finite for any $u \in X$.

The following lemma justifies the interpretation of $X$ as connecting orbits between critical points of $I$.

LEMMA 3.2. Assume that (H1)-(H4) and (H) are satisfied. Let $u \in X$ and $c_{+}=\lim _{s \rightarrow+\infty} I(u(s))<\infty$. Then $u(s) \rightarrow_{s \rightarrow+\infty} u_{+\infty}$ in the $L^{2}(\mathbb{R})$ topology, and $u_{+\infty} \in$ $H^{1,2}, I\left(u_{+\infty}\right)=c_{+}, I^{\prime}\left(u_{+\infty}\right)=0$. The same is true when one replaces $+\infty$ by $-\infty$.

Proof. For simplicity, we just consider the case $s \rightarrow+\infty$. First we notice that $\left\|\partial_{s} u(s, \cdot)\right\|_{L^{2}(d t)}^{2}>0$ for all $s \in \mathbf{R}$, if not $u \equiv x_{0}$.

Otherwise, we would have $\partial_{s} u\left(s_{0}, t\right)=0$ for some $s_{0}$ and all $t \in \mathbb{R}$. Then we could define

$$
v(s, t):=\left\{\begin{array}{l}
u(s, t): s \leqslant s_{0} \\
u\left(s_{0}, t\right): s_{0} \leqslant s \leqslant s_{0}+1 \\
u(s-1, t): s \geqslant s_{0}+1
\end{array}\right.
$$


The $v$ thus defined is in $\mathscr{E}$ and satisfies $\bar{\partial} v+H^{\prime}(t, v)=0$. Moreover, $v$ agrees with $w(s, t):=u\left(s_{0}, t\right)$ on an open subset of $\mathbb{R}^{2}$, and $\bar{\partial} w+H^{\prime}(w)=0$. From Aronszajn's unique continuation theorem ([A]; see also [H], Lemma 4), it follows that $v \equiv w$, and hence $u(s, t)=u\left(s_{0}, t\right)$ for all $s \in \mathbb{R}$, hence $u \equiv x_{0}$. If $u \equiv x_{0}$ the proposition holds true. So we may assume $u \neq \equiv x_{0}$.

Then we can make the change of variables $d \sigma=\left\|\partial_{s} u\right\|_{L^{2}(d t)}^{2} d s, \sigma(0)=0$, and define $L=\lim _{s \rightarrow+\infty} \sigma(s), \tilde{u}(\sigma)=u(s(\sigma), \cdot)$. $\tilde{u}(\sigma)$ satisfies $(\partial / \partial \sigma) \tilde{u}=\left(I^{\prime}(\tilde{u})\right) /\left(\left\|I^{\prime}(u)\right\|_{L^{2}}^{2}\right)=V(\tilde{u})$. Note that $L=\int_{0}^{\infty}\left|\partial_{s} u\right|^{2} d s d t<+\infty$.

First case. $\int_{0}^{L}\|V(\tilde{u})\|_{L^{2}} d \sigma<+\infty$.

Then $\tilde{u}(\sigma) \rightarrow_{\sigma \rightarrow L} u_{\infty}$, in the $L^{2}$-topology. Moreover, there is a subsequence $s_{n} \rightarrow$ $+\infty$ such that $I^{\prime}\left(u\left(s_{n}, \cdot\right)\right) \rightarrow 0$ in $L^{2}$ norm, because $\int d s d t\left|\partial_{s} u\right|^{2}<+\infty$. Moreover, $I\left(u\left(s_{n}, \cdot\right)\right) \rightarrow c$.

So, applying Proposition 2.5, we find

$$
\left\|\tilde{u}\left(\sigma\left(s_{n}\right)\right)-\bar{p}_{n} * \bar{u}\right\|_{H^{1,2}} \rightarrow 0
$$

after extraction. We have then

$$
\left\|u_{\infty}-\bar{p}_{n} * \bar{u}\right\|_{L^{2}} \rightarrow 0
$$

and hence $\bar{p}_{n} * \bar{u}=u_{\infty}$ for each $n$ large enough, and $u_{\infty}$ is a critical point, with $I_{\infty}(u)=c$.

Second case. $\int_{0}^{L}\|V(\tilde{u})\|_{L^{2}} d \sigma=+\infty$.

By the same arguments as in the proof of Lemma 5.2 in [CZES], we find a sequence $\sigma_{n} \rightarrow L$, with $\int_{\sigma_{n}}^{\sigma_{n+1}}\|V(\tilde{u})\|_{L^{2}} d \sigma \rightarrow 0$ (hence $\left\|\tilde{u}\left(\sigma_{n+1}\right)-\tilde{u}\left(\sigma_{n}\right)\right\|_{L^{2}} \rightarrow 0$ ), and

$$
\left\|I^{\prime}\left(\tilde{u}\left(\sigma_{n}\right)\right)\right\|_{L^{2}} \rightarrow 0, I\left(\tilde{u}\left(\sigma_{n}\right)\right) \rightarrow c .
$$

So, applying Proposition 2.8, we find $\tilde{u}\left(\sigma_{n}\right) \rightarrow u_{\infty}$ in $H^{1,2}$, and $u_{\infty}$ is a critical point of $I$ with $I\left(u_{\infty}\right)=c$. Now, for $\sigma \in\left[\sigma_{n}, \sigma_{n+1}\right]$,

$$
\begin{aligned}
\left\|\tilde{u}(\sigma)-u_{\infty}\right\|_{L^{2}} & \leqslant\left\|\tilde{u}(\sigma)-\tilde{u}\left(\sigma_{n}\right)\right\|_{L^{2}}+\left\|\tilde{u}\left(\sigma_{n}\right)-u_{\infty}\right\|_{L^{2}} \\
& \leqslant \int_{\sigma_{n}}^{\sigma}\|V(\tilde{u})\|_{L^{2}}+\left\|\tilde{u}\left(\sigma_{n}\right)-u_{\infty}\right\|_{L^{2}} \underset{n \rightarrow+\infty}{\longrightarrow} 0 .
\end{aligned}
$$

Because of the previous lemma we can unambiguously associate to each $u \in X$ its limiting orbits $u_{ \pm \infty}=L^{2}-\lim _{s \rightarrow \pm \infty} u(s)$. From Lemma 2.2 ,

$$
I\left(u_{-\infty}\right) \geqslant \frac{1}{K}\left\|u_{-\infty}\right\|_{B^{1,2}}^{2} \geqslant 0
$$

Therefore $I(u(s)) \geqslant 0$ for all $u \in X$ and $s \in \mathbb{R}$. 
Further, we can define for $x_{*} \in \mathscr{C}$

$$
X\left(x_{*}\right):=\left\{u \in X \mid u_{+\infty}=x_{*}\right\}
$$

We have

$$
X=\bigcup_{x_{*} \in \varphi} X\left(x_{*}\right)
$$

For $u \in X_{\bar{q}, R}$, the boundary conditions imply

$$
I(u(0))=-\int_{\mathbb{R}} H(t, q, 0) d t \geqslant 0
$$

and

$$
\begin{aligned}
I(u(R)) & =\int_{-T}^{T}[\langle\dot{\bar{q}}(t), p(R, t)\rangle-H(u(R, t))] d t \\
& \leqslant \int_{-T}^{T}\left[|\dot{q}(t) \| p(R, t)|-\frac{1}{2} c_{3}|p(R, t)|^{2}+c_{5}\right] d t \\
& \leqslant c\left(\|\dot{\bar{q}}\|_{C^{0}}\right) .
\end{aligned}
$$

Here we have used the condition $\langle\partial H / \partial p, p\rangle \geqslant c_{3}|p|^{2}$ for $|p| \geqslant p_{0}, c_{3}, p_{0}>0$ (H4), to deduce $H \geqslant(1 / 2) c_{3}|p|^{2}-c_{5}$ by integration along the fibre.

Combining these results with Lemma 3.1 we see that the norms $\|\cdot\|_{\text {semiloc }}^{1,2}$ defined at the beginning of this section are dominated in $X\left(x_{*}\right)$ and $X_{\bar{q}, R}$ by ${ }_{\text {sem }}\left(x_{*}\right)$ and $\|\dot{\bar{q}}\|_{c^{\circ}}$ respectively.

The following lemmata extend this result to other norms.

LEMMA 3.3. (i) If (H1)-(H4) are satisfied, then

$$
\|u\|_{C^{\circ}} \leqslant c\left(\|\dot{\bar{q}}\|_{C^{\circ}}\right) \text { for } u \in X_{\bar{q}, R},
$$

independently of $\boldsymbol{R}$.

(ii) If, moreover, $\mathscr{C} / \mathbf{Z}$ is finite, then, for $x_{*} \in \mathscr{C}$,

$$
\|u\|_{c^{\circ}} \leqslant c\left(I\left(x_{*}\right)\right) \text { for } u \in X\left(x_{*}\right) \text {. }
$$

Proof. We shall omit some steps in the proof that can be found in [Ci], Chapter 4. Denoting by $\Delta=\partial_{s}^{2}+\partial_{t}^{2}$ the Laplacian, we calculate using assumption (H4)

$$
(\Delta+\mu)|p|^{2} \geqslant-\mu,
$$

where $p$ is the fibre part of $u \in X_{\bar{q}, R}$ or $X\left(x^{*}\right)$ and $\mu>0$ is some constant. 
Then we cut $Z_{R}$ (respectively $\mathbb{R}^{2}$ ) into narrow strips of the form $Z_{\varepsilon}=\left[s_{0}-\varepsilon\right.$, $\left.s_{0}+\varepsilon\right] \times \mathbb{R}$ with $\varepsilon>0$ arbitrarily small, in particular $1 / \varepsilon^{2}>\mu$, and such that $\left\|I^{\prime}\left(u\left(s_{0} \pm \varepsilon\right)\right)\right\|_{L^{2}(d t)} \leqslant C\left(\varepsilon,\left\|\partial_{s} u\right\|_{L^{2}(d s d t)}\right)$ if $s_{0} \pm \varepsilon \notin\{0, R\}$.

We will first treat the case $s_{0}-\varepsilon \notin\{0, R\}$ and $s_{0}+\varepsilon \notin\{0, R\}$. Then from Lemma 2.2 we get a $C^{0}$ estimate on $\partial Z_{\varepsilon}$. Let

$$
v:=\sup _{\partial Z_{z}}|p|^{2}
$$

Choose a bounded domain $\mathscr{U} \subset Z_{\varepsilon}$ with smooth boundary and such that $|p|^{2}<v$ in $Z_{\varepsilon} \backslash \mathscr{U}$. Define $v \in C^{\infty}(\mathscr{U})$ by

$$
v(s, t):=\mu(v+1)\left(\varepsilon^{2}-\left(s-s_{0}\right)^{2}\right)+v .
$$

One calculates

$$
\begin{cases}(-\Delta-\mu)\left(v-|p|^{2}\right) \geqslant 0 & \text { in } \mathscr{U} \\ \left(v-|p|^{2}\right) \geqslant 0 & \text { on } \partial \mathscr{U} .\end{cases}
$$

Now the spectrum of the operator $-\Delta: L^{2}\left(Z_{\varepsilon}\right) \supset H_{0}^{2,2}\left(Z_{\varepsilon}\right) \rightarrow L^{2}\left(Z_{\varepsilon}\right)$ (where $H_{0}^{2,2}$ is the closure of $C_{0}^{\infty}$ in the $H^{2,2}$ norm) is easily seen to be $\left[\pi^{2} / 4 \varepsilon^{2}, \infty\right)$. Because of monotonicity, the smallest eigenvalue $\lambda_{1}(\mathscr{Q})$ of $-\Delta$ on $\mathscr{Q}$ with Dirichlet boundary conditions satisfies

$$
\lambda_{1}(\mathscr{U}) \geqslant \frac{\pi^{2}}{4 \varepsilon^{2}}>\mu
$$

By the refined maximum principle of $[\mathrm{Am}]$, Theorem 4.3, and Theorem 4.4,

$$
v-|p|^{2} \geqslant 0 \text { in } \mathscr{U} \text {. }
$$

This gives us a bound for $|p|^{2}$ on $Z_{\varepsilon}$.

If $s_{0}-\varepsilon=0$, then $\left|p\left(s_{0}-\varepsilon\right)\right|=0$, and the proof works as before. So it only remains to treat the case $s_{0}+\varepsilon=R$ and the maximum $v=\sup _{a z_{2}}|p|^{2}$ is attained in some $z=(R, t)$. Of course we may assume that $v \geqslant p_{0}^{2}$ with the constant $p_{0}$ of (H4).

Again it follows that $|p|^{2}$ in $Z_{\varepsilon}$ is estimated by a constant depending on $v$. However, in this case we do not yet have an a priori bound for $v$. Therefore we calculate (with $v$ defined as before, $K: T T M \rightarrow T M$ the Levi-Civita connection, and $\nabla$ the covariant derivative)

$$
0 \geqslant \partial_{s}\left(v-|p|^{2}\right)(z)=2\left[-\left\langle p, \nabla_{s} p\right\rangle-\mu(v+1) \varepsilon\right] .
$$

Using the differential equation $\nabla_{s} p=\partial_{t} q-K H^{\prime}(t, u)$ and the assumption $\langle p$, 
$\left.K H^{\prime}(t, u)\right\rangle \geqslant c_{3}|p|^{2}$ for $|p| \geqslant p_{0}$, we obtain

$$
0 \geqslant 2\left[-|p(z)||\dot{q}(t)|+c_{3}|p(z)|^{2}-\mu \varepsilon\left(|p(z)|^{2}+1\right)\right] .
$$

Having chosen $\varepsilon$ sufficiently small this implies

$$
v=|p(z)|^{2} \leqslant c\left(\|\dot{\bar{q}}\|_{c^{0}}\right) \text {. }
$$

LEMMA 3.4. (i) Assuming that (H1)-(H4) are satisfied, for $\bar{q}$ in a compact subset $K$ of $\Omega_{T}^{\infty}, X_{\bar{q}, R}$ is bounded in $C^{\infty}$ by constants depending on $K$ but independent of $\bar{q}, R$.

(ii) If, moreover, $\mathscr{C} / \mathbf{Z}$ is finite, for $x_{*} \in \mathscr{C}, X\left(x_{*}\right)$ is bounded in $C^{\infty}$.

Proof. Analogous to [Ci], Chapter 5. First assume that there would exist sequences $u_{n} \in X_{\bar{q}, R_{n}}$ and $z_{n} \in Z_{R_{n}}$ such that the gradient $\left|\nabla u_{n}\left(z_{n}\right)\right| \rightarrow \infty$. By a wellknown procedure this would lead to the "bubbling off" of a holomorphic sphere or disk with certain boundary conditions, which both cannot occur for topological reasons. So we must have a $C^{1}$-bound on $X_{\bar{q}, R}$. By the Ascoli-Arzela theorem, $X_{\bar{q}, R}$ is compact in the $C^{0}$ topology. Therefore we can localize around finitely many $u^{i} \in X_{\bar{q}, R}$ and use the local estimates for the Cauchy-Riemann operator to bootstrap up to $C^{\infty}$.

The following lemma gives an estimate on the behavior of $u(s, t)$ as $|t| \rightarrow \infty$ for $u \in X_{\bar{q}, R}$. It is inspired by [HW], Lemma 4.4 .

LEMMA 3.5. Assuming that (H1)-(H4) are satisfied, for $\bar{q}$ in a compact subset $K$ of $\Omega_{T}^{\infty}$ and $R, \varepsilon>0$, there is $D(\varepsilon, K, R) \geqslant T$ such that

$$
\|u\|_{C^{0}([0, R] \times R \backslash[-D, D])} \leqslant \varepsilon, \quad \forall u \in X_{\bar{q}, R} .
$$

Proof. Assume by contradiction that $u_{n} \in X_{\bar{q}_{n}, R}, \bar{q}_{n} \in K,\left|u_{n}\left(s_{n}, t_{n}\right)\right| \geqslant \varepsilon_{0},\left|t_{n}\right| \rightarrow$ $\infty$. Let $v_{n}(s, t)=u_{n}\left(s, t+\left[t_{n}\right]\right)$, where $\left[t_{n}\right] \in \mathbf{Z},\left[t_{n}\right] \leqslant t_{n}<\left[t_{n}\right]+1$. Then $\bar{\partial} v_{n}+$ $H^{\prime}\left(t, v_{n}\right)=0,\left|v_{m}\left(s_{n}, t_{n}-\left[t_{n}\right]\right)\right| \geqslant \varepsilon, \tau v_{n}(R, \cdot) \rightarrow q_{0}$, and $(d / d t) \tau v_{n}(R, \cdot) \rightarrow 0$ uniformly on compact subsets of $\mathbb{R}$. Moreover, $\left\|v_{n}\right\|_{B^{1,2}\left(Z_{n}\right)}$ is bounded independently of $n$, from Lemma 3.1.

By Lemma 3.4 and the Ascoli-Arzela theorem, after extraction, for any $\alpha \in \mathbb{N}^{2}$, we have $\partial^{\alpha} v_{n} \rightarrow \partial^{\alpha} v_{\infty}$ uniformly on compact sets. Here, $v_{\infty}$ is in $C^{\infty} \cap H^{1,2}\left(Z_{R}\right)$ and $\tau v_{\infty}(R, \cdot)=q_{0}, v_{\infty}(0, \cdot) \in M$. So, from $(\mathrm{H} 1), I\left(v_{\infty}(0)\right) \geqslant 0$ and $I\left(v_{\infty}(R)\right) \leqslant 0$.

But $\left|v_{\infty}\left(s_{\infty}, r_{\infty}\right)\right| \geqslant \varepsilon$, where $\left(s_{\infty}, r_{\infty}\right)$ is a limit point of $\left(s_{n}, t_{n}-\left[t_{n}\right]\right)$, and hence $v_{\infty} \neq x_{0}$ and $\int_{z_{k}}\left|\partial_{s} v_{\infty}\right|^{2} d s d t>0$. Moreover, $\bar{\partial} v_{\infty}+H^{\prime}\left(t, v_{\infty}\right)=0$ so that

$$
\left\|\partial_{s} v_{\infty}\right\|_{L^{2}\left(Z_{x}\right)}^{2}=I\left(v_{\infty}(R)\right)-I\left(v_{\infty}(0)\right)=0
$$

So there is a contradiction, and the lemma is proved.

In fact, $u(s, t)$ decreases exponentially as $|t|$ tends to infinity. 
Lemma 3.6. Assume that (H1)-(H4) are satisfied. Let $\alpha \in \mathbb{N}^{2}, R>0, K \subset \Omega_{T}^{\infty}$ a compact set. Then there are $C, \rho>0$ depending only on $\alpha, R, K$ and such that

$$
\left|\partial^{\alpha} u(s, t)\right| \leqslant C e^{-\rho|t|}, \quad \forall u \in \bigcup_{\bar{q} \in \mathbf{K}} X_{\bar{q}, R} .
$$

Proof. For simplicity, we just consider the case $t>0$. Take a smooth chart $\phi: \mathscr{Q} \subset \mathbb{R}^{2 n} \rightarrow B\left(x_{0}, \varepsilon_{0}\right)$ and consider $D \geqslant T$ such that $|u(s, t)| \leqslant \varepsilon_{0}$ for any $u \in$ $\bigcup_{\bar{q} \in \mathbf{K}} X_{\bar{q}, R}$ and any $t \geqslant D$. Then, in local coordinates, for $t \geqslant D$.

$$
\bar{\partial} u+\mathscr{A}(t) u=-R^{\prime}(t, u)
$$

with $R(t, u)=H(t, u)-(1 / 2)\langle u \mathscr{A}(t) u\rangle$.

Take $\theta \in C^{\infty}(\mathbb{R},[0,1])$ such that $\theta=0$ on $\mathbb{R}_{-}, \theta=1$ on $[1,+\infty)$. For $\Delta>0$, $n \in \mathbb{N}$ such that $n \Delta \geqslant D$, we define $v_{n, \Delta}=\theta(t / \Delta-n) u$, which can be idendified with an element of $T_{x_{0}} \mathscr{E}_{q_{0}, R}$, since $D \geqslant T$. Then

$$
\bar{\partial} v_{n, \Delta}+\mathscr{A}(t) v_{n, \Delta}=-\theta\left(\frac{t}{\Delta}-n\right) R^{\prime}(t, u)+J(u) \frac{\theta\left(\frac{t}{\Delta}-n\right)}{\Delta} u .
$$

From the proof of Lemma 4.2,

$$
\|v\|_{H^{1,2}} \leqslant c\left\|\left(\partial_{s}+J\left(x_{0}\right) \partial_{t}+\mathscr{A}(t)\right) v\right\|_{L^{2}}
$$

for all $v \in T_{x_{0}} \mathscr{E}_{q_{0}, R}$. So

$$
\begin{aligned}
\|u\|_{H^{1,2}([0, R] \times[(n+1) \Delta, \infty))} \leqslant & \left\|v_{n, \Delta}\right\|_{B^{1,2}\left(Z_{n}, R^{2 n}\right)} \\
\leqslant & c\left(\left\|J(u)-J\left(x_{0}\right)\right\|_{C^{0}([0, R] \times[n \Delta,+\infty))}\left\|\partial_{t} u\right\|_{L^{2}([0, R] \times[n \Delta,+\infty \|)}\right. \\
& +\left\|\frac{R^{\prime}(t, u)}{|u|}\right\|_{C^{0}([0, R] \times[n \Delta,+\infty))}\|u\|_{L^{2}([0, R] \times[n \Delta,+\infty))} \\
& \left.+\frac{1}{\Delta}\|u\|_{L^{2}([0, R] \times[n \Delta,+\infty))}\right) \\
\leqslant & \frac{1}{2}\|u\|_{B^{1,2}([0, R] \times[n \Delta,+\infty))}
\end{aligned}
$$

for $\Delta$ fixed large enough, and any $n \geqslant 1, u \in \bigcup_{\bar{q} \in \mathbb{R}} X_{\bar{q}, R}$. 
As a consequence,

$$
\|u\|_{H^{1,2}([0, R] \times[t,+\infty))} \leqslant C_{0} e^{-\rho_{0} t}
$$

for some $C_{0}, \rho_{0}>0$ and for any $t \geqslant 0$. Combining this with Lemma 3.4, one easily ends the proof of Lemma 3.6.

The following result is an immediate consequence of Lemma 3.6.

Proposition 3.7. Assume that (H1)-(H4) are satisfied. Given $R>0$ and $K \subset$ $\Omega_{T}^{\infty}$ compact, the set $\bigcup_{\bar{q} \in K} X_{\bar{q}, R}$ is compact in $H^{2,2}\left(Z_{R}, T M\right)$.

We now want the estimates above to become independent of $R$. This is possible if $\mathscr{C} / \mathbf{Z}$ is finite.

LEMMA 3.8. Assume that (H1)-(H4) and ( $\mathscr{H})$ are satisfied. Then:

(i) For $\bar{q}$ in a compact subset $K$ of $\Omega_{T}^{\infty}$ and $\varepsilon>0$, there is a $D(\varepsilon, K) \geqslant T$ such that for any $R \geqslant 1$ and $u \in X_{\bar{q}, R}$,

$$
\|u\|_{C^{\circ}([0, R] \times \mathbf{R} \backslash[-D, D])} \leqslant \varepsilon .
$$

(ii) Given $x_{*} \in \mathscr{C}$ and $\varepsilon>0$, there is a $D\left(\varepsilon, x_{*}\right)>0$ such that, for any $u \in X\left(x_{*}\right)$,

$$
\|u\|_{C^{0}(\mathbf{R} \times \mathbf{R} \backslash[-D, D])} \leqslant \varepsilon .
$$

Proof. The proofs of (i) and (ii) are similar, so we just give the proof of (ii).

First step. Given $\Delta, \beta>0$ and $u \in X\left(x_{*}\right)$, we find a partition of $\mathbb{R}$ by $2 N+1$ intervals $I_{0}, \ldots, I_{2 N}, N \leqslant N_{0}, N_{0}$ depending only on $x_{*}$, with $I_{0}=\left(-\infty, S_{1}\right]$, $I_{k}=\left[S_{k}, S_{k+1}\right]$ for $1 \leqslant k \leqslant 2 N-1, I_{2 N}=\left[S_{2 N},+\infty\right)$, and such that:

(a) For $k=2 q$, there is $Y_{q}=\bar{p}_{q} * \bar{x}_{q}$ with $x_{q}^{i} \in \mathscr{P}, p_{q}^{i} \in \mathbf{Z}, p_{q}^{i+1}-p_{q}^{i} \geqslant \Delta$, and such that

$$
\begin{aligned}
\left\|u(s, \cdot)-Y_{q}\right\|_{L^{2}(d t)} \leqslant \beta & \text { for any } s \in I_{k}, \\
\left\|u\left(S_{k}, \cdot\right)-Y_{q}\right\|_{H^{1,2}(d t)} \leqslant \beta & \text { for } 1 \leqslant k \leqslant 2 N, \\
\left\|u\left(S_{k+1}, \cdot\right)-Y_{q}\right\|_{H^{1,2}(d t)} \leqslant \beta & \text { for } 0 \leqslant k \leqslant 2 N-1 .
\end{aligned}
$$

(b) There is $L(\Delta, \beta)$ such that, for $k=2 q-1$,

$$
0<S_{2 q}-S_{2 q-1}<L .
$$

Proof of the first step. Given $L>0$, in each interval $[n(L / 2),(n+1)(L / 2)]$, $n \in \mathbf{Z}$, there is an $s_{n}$ such that

$$
\left\|I^{\prime}\left(u\left(s_{n}\right)\right)\right\|_{L^{2}(d t)}^{2} \leqslant \frac{2 I\left(x_{*}\right)}{L}
$$


So, from Corollary 2.6, given $\Delta, \alpha>0$ and $u \in X\left(x_{*}\right)$, there are $L(\Delta, \alpha)>0$ and a sequence $\left(s_{n}\right)_{n \in \mathbf{Z}}$ such that $0<s_{n+1}-s_{n}<L$ and $\left\|u\left(s_{n}, \cdot\right)-\bar{p}_{n} * \bar{x}_{n}\right\|_{H^{1.2}(d t)} \leqslant \alpha$ for some $\bar{p}_{n} \in \mathbf{Z}^{m(n)}, \bar{x}_{n} \in \mathscr{P}^{m(n)}$, with $p_{n}^{i+1}-p_{n}^{i} \geqslant \Delta$, for all $i$, and $m(n)$ bounded because $I\left(u\left(s_{n}\right)\right) \leqslant I\left(x_{*}\right)$.

We recall that $\mathscr{P} \subset \mathscr{C}$ is a finite set such that $\mathscr{B} \backslash\left\{x_{0}\right\}=\mathbf{Z} * \mathscr{P}$. From Lemma 2.7 , there are $\Delta_{0}, \alpha_{0}$ such that, if $\bar{p}_{n} * \bar{x}_{n}$ and $\bar{p}_{n+1} * \bar{x}_{n+1}$ are not the same, then

$$
\int_{s_{n}}^{s_{n+1}}\left\|\partial_{s} u\right\|_{L^{2}(d t)}^{2} d s \geqslant\left(r_{0}-\alpha_{0}\right) \mu_{0}>0,
$$

where $r_{0}$ has been defined in Lemma 2.7, and

$$
\begin{aligned}
\mu_{0}= & \inf \left\{\left\|I^{\prime}(x)\right\|_{L^{2}} \mid x \in B_{L^{2}}\left(\bar{k} * \bar{y}, r_{0}\right) \backslash B_{L^{2}}\left(\bar{k} * \bar{y}, \alpha_{0}\right), \bar{y} \in \mathscr{P}^{m}, m \geqslant 1,\right. \\
\bar{k} & \left.\in \mathbf{Z}^{m}, k^{i+1}-k^{i} \geqslant \Delta_{0}\right\}
\end{aligned}
$$

So

$$
\begin{aligned}
\operatorname{Card}\left\{n \in \mathbf{Z} \mid \bar{p}_{n} * \bar{x}_{n} \neq \bar{p}_{n+1} * \bar{x}_{n+1}\right\} & \leqslant \frac{\int_{R^{2}}\left|\partial_{s} u\right|^{2}}{\left(r_{0}-\alpha_{0}\right) \mu_{0}} \\
& \leqslant \frac{I\left(x_{*}\right)}{\left(r_{0}-\alpha_{0}\right) \mu_{0}} \leqslant N_{0}
\end{aligned}
$$

for some $N_{0} \in \mathbb{N}$.

On the other hand, if $\bar{p}_{n} * \bar{x}_{n}=\bar{p}_{n+1} * \bar{x}_{n+1}$, then, for $\Delta \geqslant \Delta(\beta)$ and $\alpha \leqslant \alpha(\beta)$, we have

$$
\left\|u(s, \cdot)-\bar{p}_{n} * \bar{x}_{n}\right\|_{L^{2}(d)} \leqslant \beta, \quad \forall s \in\left[s_{n}, s_{n+1}\right] .
$$

The explanation is the following: We define

$$
\begin{aligned}
\mu(\beta)= & \inf \left\{\left\|I^{\prime}(x)\right\|_{L^{2}} \mid x \in B_{L^{2}}(\bar{k} * \bar{y}, \beta) \backslash B_{L^{2}}\left(\bar{k} * \bar{y}, \frac{\beta}{2}\right), \bar{y} \in \mathscr{P}^{m}, m \geqslant 1,\right. \\
& \left.\bar{k} \in \mathbf{Z}^{m}, k^{i+1}-k^{i} \geqslant \Delta(\beta)\right\} \\
> & 0 .
\end{aligned}
$$

Assume by contradiction that, for some $s \in\left[s_{n}, s_{n+1}\right]$,

$$
\left\|u(s, \cdot)-\bar{p}_{n} * \bar{x}_{n}\right\|_{L^{2}} \geqslant \beta \text {. }
$$


Then

$$
\int_{s_{n}}^{s_{n+1}}\left\|\partial_{s} u\right\|_{L^{2}(d t)}^{2} \geqslant \frac{\beta}{2} \mu(\beta)
$$

provided $\alpha \leqslant \beta / 2$. So $I\left(u\left(s_{n+1}\right)\right)-I\left(u\left(s_{n}\right)\right) \geqslant(\beta \mu(\beta)) / 2$, which is impossible for $\alpha \leqslant \alpha(\beta)$.

The existence of the partition $I_{0}, \ldots, I_{2 N}$ easily follows from the above arguments.

Second step. We consider the partition $I_{0}, \ldots, I_{2 N}$ of the first step. We are going to prove, by induction on $q \leqslant N_{0}$, that for $\Delta=\Delta^{0}$ fixed and large enough, and $0<\beta \leqslant \beta^{0}$ with $\beta^{0}$ small enough,

(1) $Y_{N-q} \in \mathscr{F}_{q}$, where $\mathscr{F}_{q}$ is a finite subset of $\mathscr{C}$ depending only on $q, x_{*}$.

(2) For $x_{*} \in \mathscr{C}, q \leqslant N-1$ fixed, there are two functions $\varepsilon(\beta)$ and $D(\beta)$ such that

$$
\|u\|_{C^{0}\left(I_{2(N-q)-1} \times R \backslash[-D, D]\right)} \leqslant \varepsilon,
$$

with $\lim _{\beta \rightarrow 0} \varepsilon(\beta)=0$.

The same is true for $x_{*} \in \mathscr{C}, q \leqslant N$ fixed, with $I_{2(N-q)}$ instead of $I_{2(N-q)-1}$. Since $N$ is bounded independently of $\beta$, Lemma 3.8 is an immediate consequence of (2).

Proof of the second step. If we take $\Delta^{0}$ large enough and $\beta^{0}$ small enough, it follows from Lemma 2.7 and Lemma 3.2 that $Y_{N}=x_{*}$. Then from (a) of the first step and Lemma 3.4, we find easily the estimate

$$
\|u\|_{C^{\circ}\left(I_{2 N} \times R \backslash\left[-D_{0}(\beta), D_{0}(\beta)\right]\right)} \leqslant \varepsilon_{0}(\beta)
$$

with $\lim _{\beta \rightarrow 0} \varepsilon_{0}(\beta)=0$.

Let us prove that there are $\varepsilon_{1}(\beta)$ and $D_{1}(\beta)$ such that

$$
\|u\|_{C^{0}\left(I_{2 N-1} \times R \backslash\left[-D_{1}, D_{1}\right]\right)} \leqslant \varepsilon_{1},
$$

with $\lim _{\beta \rightarrow 0} \varepsilon_{1}(\beta)=0$. Otherwise, for some $\hat{\varepsilon}>0$ and for any $\beta>0$, there would exist sequences $u_{n} \in X\left(x_{*}\right)$ and $\left(s_{n}, t_{n}\right) \in I_{2 N-1} \times \mathbb{R}$ such that $\left|t_{n}\right| \rightarrow \infty$ and $\left|u_{n}\left(s_{n}, t_{n}\right)\right|$ $\geqslant \varepsilon$.

Denoting $v_{n}(s, t)=u_{n}\left(s+s_{n}, t+\left[t_{n}\right]\right)$ as in the proof of Lemma 3.5, we find after extraction $\partial^{\alpha} v_{n} \rightarrow \partial^{\alpha} v_{\infty}$, uniformly on compact sets, for any $\alpha \in \mathbb{N}^{2}$. Moreover,

$$
\begin{aligned}
S_{2 N}-S_{n} & \rightarrow b \\
S_{2 N-1}-S_{n} & \rightarrow a,
\end{aligned}
$$


$v_{\infty}$ is in $C^{\infty} \cap H_{\text {semiloc }}^{1,2}\left(\mathbb{R}^{2}\right)$ and satisfies

$$
\begin{aligned}
& \text { (v1) } \bar{\partial} v_{\infty}+H^{\prime}\left(t, v_{\infty}\right)=0 \\
& \text { (v2) }(\forall s \geqslant b) \quad\left\|v_{\infty}(s, \cdot)\right\|_{L^{2}(d t)} \leqslant \beta, \\
& \text { (v3) } v_{\infty}(0, \cdot) \neq x_{0}, \\
& \text { (v4) } \int_{\mathbb{R}^{2}}\left|\partial_{s} v_{\infty}\right|^{2}<\infty .
\end{aligned}
$$

From Lemma 2.3 and Proposition 3.2, if $\beta<\rho$, then $u(s, \cdot) \overrightarrow{L^{2}} x_{0}$ as $s \rightarrow+\infty$. So Proposition 3.2 gives also $x_{-}=x_{0}$ and $u=x_{0}$, and we have found a contradiction. Therefore $\varepsilon_{1}(\beta)$ and $D_{1}(\beta)$ exist. For $\beta$ small enough, this gives the existence of a finite set $\mathscr{F}_{1} \subset \mathscr{C}$ independent of $\beta$ and such that $Y_{q-1} \in \mathscr{F}_{1}$.

Repeating the same arguments at most $N_{0}$ times, we finally prove the second step and Lemma 3.8 . lines.

We now give the analogue of Lemma 3.6 for large values of $R$ and infinite flow

Proposition 3.9. Assume that $(\mathrm{H} 1)-(\mathrm{H} 4)$ and $(\mathscr{H})$ are satisfied. Then:

(i) Given a compact subset $K$ of $\Omega_{T}^{\infty}$ and $\alpha \in \mathbb{N}^{2}$, there are $C, \rho>0$ such that for any $R \geqslant 1$ and $u \in \bigcup_{\bar{q} \in K} X_{\bar{q}, R}$,

$$
\left|\partial^{\alpha} u(s, t)\right| \leqslant C e^{-\rho|t|} .
$$

(ii) Given $x_{*} \in \mathscr{C}$ and $\alpha \in \mathbb{N}^{2}$, there are $C, \rho>0$ such that, for any $u \in X\left(x_{*}\right)$,

$$
\left|\partial^{\alpha} u(s, t)\right| \leqslant C e^{-\rho|z|}
$$

Proof. We just prove (ii), for $t \geqslant 0$. We take the same smooth chart $\phi: q \subset$ $\mathbb{R}^{2 n} \rightarrow B\left(x_{0}, \varepsilon_{0}\right)$ as in the proof of Lemma 3.6.

Consider $\Theta \in C^{\infty}\left(\mathbb{R}^{2},[0,1]\right)$ such that

$$
\begin{array}{ll}
\Theta(s, t)=0 & \text { on }\left(\mathbf{R} \times \mathbf{R}_{-}\right) \cup((\mathbf{R} \backslash[-2,2]) \times \mathbf{R}), \\
\Theta(s, t)=1 & \text { on }[-1,1] \times[1,+\infty) .
\end{array}
$$

For $\Delta>0,(\sigma, n) \in \mathbb{R} \times \mathbb{N}$ with $n \Delta$ large enough, we define in local coordinates $V_{\sigma, n, \Delta}=\Theta((s-\sigma) / \Delta,(t / \Delta)-n) u(s, t)$, which can be idendified with an element of $T_{x_{0}} \mathscr{E}_{q_{0}, R}$. 
Denoting by $\Theta_{s}, \Theta_{t}$ the partial derivatives of $\Theta$ with respect to $s, t$, we have

$$
\begin{aligned}
\bar{\partial} V_{\sigma, n, \Delta}+\mathscr{A}(t) V_{\sigma, n, \Delta}= & -\Theta\left(\frac{s-\sigma}{\Delta}, \frac{t}{\Delta}-n\right) R^{\prime}(t, u) \\
& +\frac{\Theta_{s}\left(\frac{s-\sigma}{\Delta}, \frac{t}{\Delta}-n\right)+\Theta_{t}\left(\frac{s-\sigma}{\Delta}, \frac{t}{\Delta}-n\right) J(u)}{\Delta} u .
\end{aligned}
$$

Notice that, from the proof of Lemma 4.2,

$$
\|v\|_{H^{1,2}} \leqslant c\left\|\left(\partial_{s}+J\left(x_{0}\right) \partial_{t}+\mathscr{A}(t)\right) v\right\|_{L^{2}}
$$

for all $v \in H^{2,2}\left(\mathbb{R}^{2}, T_{x_{0}} T M\right)$.

So, by the same kind of estimates as in the proof of Lemma 3.6, we find, for $\Delta$ fixed large enough,

$$
\|u\|_{H^{1,2}([\sigma-\Delta, \sigma+\Delta] \times[(n+1) \Delta,+\infty))} \leqslant \frac{1}{4}\|u\|_{H^{1,2}([\sigma-2 \Delta, \sigma+2 \Delta] \times[n \Delta,+\infty))},
$$

and hence

$$
\sup _{\sigma \in \mathbb{R}}\|u\|_{H^{1,2}([\sigma-\Delta, \sigma+\Delta] \times[(n+1) \Delta,+\infty))} \leqslant \frac{1}{2} \sup _{\sigma \in \mathbb{R}}\|u\|_{H^{1,2}([\sigma-\Delta, \sigma+\Delta] \times[n \Delta,+\infty))} .
$$

So

$$
\sup _{\sigma \in \mathbf{R}}\|u\|_{H^{1,2}([\sigma-\Delta, \sigma+\Delta] \times[t,+\infty))} \leqslant C_{0} e^{-\rho_{0} t}
$$

and Lemma 3.4 ends the proof.

Proposition 3.9 has an immediate consequence.

Corollary 3.10. Assuming that (H1)-(H4) and ( $\mathscr{H})$ are satisfied, for $x_{*} \in \mathscr{C}$ and $m \geqslant 0, p \geqslant 1$, the set $X\left(x_{*}\right)$ is precompact in $X$ for the topology associated to $\|\cdot\|_{\text {semiloc }}$, and the set $X / Z$, quotient of $X$ by the group of integer translations in time, is compact for the quotient topology.

4. Existence of contractible flow lines. All the compactness results of the previous section would of course be useless if the only flow line was $u \equiv x_{0}$. In this section, we show that the set $X_{\bar{q}, R}$ is nonempty for any contractible $\bar{q}$. Studying the limit $R \rightarrow \infty$, we find an injection from the cohomology of the space of contractible loops containing $q_{0}$, into the cohomology of $X$. This injection will be used in Section 5, where we will prove Theorem 1.1 under the assumption " $\pi_{1}(M)$ finite." 
We begin with the set $\mathscr{E}_{\bar{q}, R}, \bar{q} \in \Omega_{T}^{\infty}, R>0$, as defined in Section 3. We have a first preliminary result.

LEMMA 4.1. $\mathscr{E}_{\bar{q}, R}$ is a Hilbert manifold, and

$$
u \mapsto \bar{\partial} u+H^{\prime}(t, u)
$$

is a smooth section in a Hilbert bundle $F_{\text {over }} \mathscr{E}_{\bar{q}, R}$ with fibres $\mathscr{F}_{u}=H^{1,2}\left(Z_{R}, u^{*} T T M\right)$.

Proof. The lemma follows from the results of [El] (Theorem 5.1, 6.1 and 6.2); see also [Ci], Lemma 3.1. The only modification needed is due to the fact that $Z_{R}$ is not compact. But if $u \in \mathscr{E}_{\bar{q}, R}$, then by Sobolev embedding $u(s, t)$ is contained in a chart around $x_{0}$ for $(s, t)$ outside some compact subset of $Z_{R}$. Thus we can trivialize and proceed as usual.

As a consequence of Kuiper's theorem, we find a smooth trivialization $\Psi: \mathscr{F} \rightarrow$ $\mathscr{E}_{\bar{q}, R} \times F$ of the bundle $\mathscr{F}$, where $F$ is the separable Hilbert space $H^{1,2}\left(Z_{R}, \mathbb{C}^{n}\right)$ (see [Pa], Chapter 9A, Corollary C6). Denoting by $\pi_{F}=\mathscr{E}_{\bar{q}, R} \times F \rightarrow F$ the projection, we can thus define a smooth map $f: \mathscr{E}_{\bar{q}, R} \rightarrow F$,

$$
f(u):=\pi_{F} \circ \Psi\left(\bar{\partial} u+H^{\prime}(t, u)\right) .
$$

Let $q_{0} \in \Omega_{T}^{\infty}$ be the constant map.

LEMMA 4.2. If $u \in \mathscr{C}_{q_{0}, R}$ and $f(u)=0$, then $u \equiv x_{0}$. Moreover, the linearization $D f\left(x_{0}\right): T_{x_{0}} \mathscr{E}_{q_{0}, R} \rightarrow F$ is an isomorphism, and the norm of its inverse is bounded independently of $R$.

Proof. (i) The boundary conditions and (H1) imply

$$
\begin{aligned}
0 & \geqslant I(u(R))-I(u(0)) \\
& =\int_{Z_{R}}\left|\partial_{s} u\right|^{2} d s d t, \text { because } f(u)=0
\end{aligned}
$$

So $u$ is independent of $s$ and therefore $u \equiv x_{0}$ from the boundary conditions.

(ii) If we identify $T_{x_{0}} T M$ with $\mathbb{C}^{n}=\mathbf{R}^{2 n}$ in the way that the horizontal subspace $T_{x_{0}}^{h} T M$ corresponds to $\mathbb{R}^{n} \subset \mathbb{C}^{n}$ and the vertical subspace $T_{x_{0}}^{v} T M$ to $J_{0} \mathbf{R}^{n}$ $\subset \mathbb{C}^{n}$, where $J_{0}=\left(\begin{array}{rr}0 & -1 \\ 1 & 0\end{array}\right)$ is the canonical complex structure on $\mathbb{C}^{n}$, then $D f\left(x_{0}\right)$ is equivalent to the operator

$$
\begin{aligned}
& g: E=\left\{u \in H^{2,2}\left(Z_{R}, \mathbb{C}^{n}\right) \mid u(0, t) \in \mathbf{R}^{n}, u(R, t) \in J_{0} \mathbb{R}^{n}\right\} \rightarrow F=H^{1.2}\left(Z_{R}, \mathbb{C}^{n}\right), \\
& g(u)=\partial_{s} u+J_{0} \partial_{t} u+\mathscr{A}(t) u
\end{aligned}
$$

Here $\mathscr{A}(t)$ is the matrix corresponding to $H^{\prime \prime}\left(t, x_{0}\right)$. 
Condition (H1) implies that the quadratic form $u \mapsto u \mathscr{A}(t) u$ is positive semidefinite on $J_{0} \mathbb{R}^{n}$ and negative semidefinite on $\mathbb{R}^{n}$.

We first prove that $g$ is injective: For $x \in H^{1,2}\left(\mathbb{R}, \mathbb{C}^{n}\right)$, we define the action integral $I_{0}(x):=\int\left(-(1 / 2)\left\langle x, J_{0} \dot{x}\right\rangle-(1 / 2)\langle x, \mathscr{A}(t) x\rangle\right) d t$ and the $L^{2}$ gradient $I_{0}^{\prime}(x)=$ $-J_{0} \dot{x}-\mathscr{A}(t) x$.

Then the boundary conditions imply, for $u \in E$,

$$
\begin{aligned}
0 & \geqslant I_{0}(u(R))-I_{0}(u(0)) \\
& =\int_{0}^{R}\left\langle\partial_{s} u(s), I_{0}^{\prime}(u(s))\right\rangle_{L^{2}(R)} d s \\
& \geqslant\left\|I_{0}^{\prime}(u)\right\|_{L^{2}\left(Z_{R}\right)}^{2}-\|g(u)\|_{L^{2}\left(Z_{R}\right)}\left\|I_{0}^{\prime}(u)\right\|_{L^{2}\left(Z_{R}\right)}
\end{aligned}
$$

Hence $\left\|I_{0}^{\prime}(u)\right\|_{L^{2}} \leqslant\|g(u)\|_{L^{2}}$, and similarly $\left\|\partial_{s} u\right\|_{L^{2}} \leqslant\|g(u)\|_{L^{2}}$. Now, by Lemma 2.1, $\|u\|_{L^{2}}+\left\|\partial_{t} u\right\|_{L^{2}} \leqslant c_{1}\left\|I_{0}^{\prime}(u)\right\|_{L^{2}}$, thus

$$
\|u\|_{H^{1,2}} \leqslant c_{2}\|g(u)\|_{L^{2}}
$$

and $c_{2}$ does not depend on $\boldsymbol{R}$.

Applying the same procedure to $\partial_{t} u$ (which satisfies the same boundary conditions as $u$ in a weak sense) gives

$$
\begin{aligned}
\left\|\partial_{t} u\right\|_{H^{1,2}} & \leqslant c_{2}\left\|g\left(\partial_{t} u\right)\right\|_{L^{2}} \\
& \leqslant c_{2}\left(\left\|\partial_{t} g(u)\right\|_{L^{2}}+\left\|\mathscr{A}^{\prime}(t) u\right\|_{L^{2}}\right) \\
& \leqslant c_{3}\|g(u)\|_{H^{1,2}}
\end{aligned}
$$

Finally,

$$
\begin{aligned}
\left\|\partial_{s} u\right\|_{B^{1,2}} & \leqslant\|g(u)\|_{H^{1,2}}+\left\|J_{0} \partial_{t} u+\mathscr{A}(t) u\right\|_{H^{1,2}} \\
& \leqslant c\|g(u)\|_{H^{1,2}}
\end{aligned}
$$

with $c$ independent of $R$.

So we have proved $\|u\|_{H^{2,2}} \leqslant c\|g(u)\|_{H^{1,2}}$, which implies the injectivity of $g$, and $g$ has a bounded left inverse $g_{l}^{-1},\left\|g_{l}^{-1}\right\| \leqslant c$.

Finally, we prove that $g$ is surjective. This will imply $g_{l}^{-1}=g^{-1}$. Let $v \in F$ be given. We approximate $v$ in the $H^{1,2}$-norm by $\left(v_{N}\right)_{N \in \mathrm{N}}$ with supp $v_{N} \subset(-N, N)$. Let

$$
E_{N}=\left\{u \in H^{2,2}\left([0, R] \times \mathbb{R} / 2 N \mathbf{Z}, \mathbb{C}^{n}\right) \mid u(0, t) \in \mathbb{R}^{n}, u(R, t) \in J_{0} \mathbb{R}^{n}\right\}
$$


The same argumentation as in (ii) shows that $g$ as an operator from $E_{N}$ to $F_{N}=$ $H^{1,2}\left([0, R] \times \mathbb{R} / 2 N Z, \mathbb{C}^{n}\right)$ is injective, and $\|u\|_{H^{2,2}} \leqslant c\|g(u)\|_{H^{1.2}}$ with a constant which is independent of $N$.

It is well known (see, e.g., [Ci], Theorem 2.3) that $\bar{\partial}: E_{N} \rightarrow F_{N}$ is an isomorphism. Since on compact domains $\mathscr{A}(t) u$ is a compact perturbation, $g: E_{N} \rightarrow F_{N}$ is a Fredholm operator of index 0 and thus an isomorphism. Therefore we find $u_{N} \in E_{N}$ with $g\left(u_{N}\right)=v_{N}$ and

$$
\left\|u_{N}\right\|_{H^{2,2}} \leqslant c\left\|v_{N}\right\|_{H^{1,2}} \text { for all } N \in \mathbf{N} \text {. }
$$

Let $\varphi_{N}(t)$ be a cut-off function which is 1 in $[-N+1, N-1]$ and 0 outside $[-N, N]$ and define

$$
\tilde{u}_{N}:=\varphi_{N}(t) u_{N} \in E
$$

Then $\left\|\tilde{u}_{N}\right\|_{H^{2,2}} \leqslant c^{\prime}$ for some constant $c^{\prime}$, and thus $\left(\tilde{u}_{N}\right)$ has a subsequence converging in $H_{\text {loc }}^{1,2}$ and weakly in $H^{2,2}$ to some $u \in E$. Since $g\left(\tilde{u}_{N}\right) \rightarrow v$ and $g\left(\tilde{u}_{N}\right) \rightarrow$ $g(u)$ in $L_{\text {loc }}^{2}, u$ satisfies $g(u)=v$.

LEMMA 4.3. If $f(u)=0$, then $D f(u)$ is a semi-Fredholm operator.

Proof. By regularity theory, $u$ is $C^{\infty}$. So we can linearize in a chart centered at $u$ and obtain (see [Ci], Proposition 3.2)

$$
D f(u) \eta=\nabla_{s} \eta+J(u) \nabla_{t} \eta+D J(u)\left(\eta, \partial_{t} u\right)+H^{n}(u) \eta,
$$

where $\eta \in H^{2,2}\left(Z_{R}, u^{*} T T M\right)$ with vertical part $\eta^{\nu}(0, t)=0$ and horizontal part $\eta^{h}(R, t)=0 . \nabla$ denotes covariant derivatives.

Now we choose a $\Delta>0$ such that $u(s, t)$ lies in a chart around $x_{0}$ for $|t| \geqslant \Delta$. Let $\varphi_{-}, \varphi_{0}, \varphi_{+}$be a partition of unity subordinate to the covering of $\mathbf{R}$ by the intervals $(-\infty,-\Delta),(-\Delta-1, \Delta+1)$ and $(\Delta, \infty)$. If we trivialize $T M$ around $q_{0}$, then $\varphi_{+} \eta$ becomes equivalent to an $\eta_{+}: Z_{\mathbb{R}} \rightarrow \mathbb{C}^{n}$, with $\eta_{+}(0, t) \in \mathbf{R}^{n}, \eta_{+}(R, t) \in$ $J_{0} \mathbb{R}^{n}$ and $\operatorname{supp}\left(\eta_{+}\right) \subset[0, R] \times(\Delta, \infty)$.

The results of Section 3 imply that all derivatives of $u$ are bounded and become arbitrarily small as $|t| \rightarrow \infty$. Together with the compact embedding $H^{2.2}\left(Z_{R}\right) \subset$ $H_{\text {loc }}^{1,2}\left(Z_{R}\right)$ this establishes that

$$
D f(u) \eta_{+}=\partial_{s} \eta_{+}+J(u) \partial_{t} \eta_{+}+\mathscr{A}(t) \eta_{+}+K_{+} \eta_{+}
$$

with a compact operator $K_{+}: H^{2,2}\left(Z_{R}\right) \rightarrow H^{1.2}\left(Z_{R}\right)$. 
From Lemma 4.2 we get

$$
\begin{aligned}
\left\|\eta_{+}\right\|_{H^{2,2}} & \leqslant c\left\|\partial_{s} \eta_{+}+J_{0} \partial_{t} \eta_{+}+A(t) \eta_{+}\right\|_{H^{1,2}} \\
& \leqslant c\left(\left\|D f(u) \eta_{+}\right\|_{H^{1,2}}+\left\|J_{0}-J(u)\right\|_{C^{1}([0, R] \times(\Delta, \infty))}\left\|\partial_{t} \eta_{+}\right\|_{H^{1,2}}+\left\|K_{+} \eta_{+}\right\|_{H^{1,2}}\right) \\
& \leqslant \frac{1}{2}\left\|\eta_{+}\right\|_{H^{2,2}}+c\left(\left\|D f(u) \eta_{+}\right\|_{H^{1,2}}+\left\|K_{+} \eta_{+}\right\|_{H^{1,2}}\right)
\end{aligned}
$$

if $\Delta$ has been chosen large enough.

An analogous estimate holds for $\varphi_{-} \eta \cdot \varphi_{0} \eta$ has its support in a compact region. Therefore the well-known local theory of the Cauchy-Riemann operator ([Ci], Lemma 2.1) provides an estimate for $\varphi_{0} \eta$.

Adding up we obtain

$$
\|\eta\|_{H^{2,2}} \leqslant c\left(\|D f(u) \eta\|_{H^{1,2}}+\|K \eta\|_{H^{1,2}}\right)
$$

with some compact operator $K: H^{2,2}\left(Z_{R}\right) \rightarrow H^{1,2}\left(Z_{R}\right)$. It is a standard result of Fredholm theory that $D f(u)$ is then a semi-Fredholm operator.

To make things a bit more complicated, we will now allow the base curves $\bar{q}$ to vary. We define

$$
\mathbf{\Omega}_{T}:=\left\{\bar{q} \in C^{0}(\mathbf{R}, M) \mid \bar{q}(t) \equiv q_{0} \text { for }|t| \geqslant T-1\right\} .
$$

Let $\mathscr{J}: \Omega_{T} \rightarrow \Omega_{T}^{\infty}$ be a smoothing operator as defined in [Ci], Chapter 3, and let

$$
\mathscr{E}_{R}:=\left\{(\bar{q}, u) \mid \vec{q} \in \Omega_{T}, u \in \mathscr{E}_{\mathscr{g}, R}\right\}
$$

with the natural projection $\pi: \mathscr{E}_{R} \rightarrow \Omega_{T},(\bar{q}, u) \mapsto \bar{q}$. It is easily seen ([Ci], Lemma 3.1) that $\mathscr{E}_{R}$ is a bundle over $\Omega_{T}$. Thus the linearizations of the operator

$$
\begin{aligned}
\pi \oplus f: \mathscr{E}_{R} & \rightarrow \Omega_{T} \times F, \\
(\bar{q}, u) & \mapsto(\bar{q}, f(u))
\end{aligned}
$$

are of the form

$$
\left(\begin{array}{cc}
1 & 0 \\
* & D f
\end{array}\right): T_{\bar{q}} \Omega_{T} \oplus T_{u} \mathscr{E}_{\bar{q}, R} \rightarrow T_{\bar{q}} \Omega_{T} \oplus F
$$

Therefore Lemmas 4.2 and 4.3 lead to analogous statements for the operator $\pi \oplus f$, which we summarize in the following corollary. 
COROLlaRY 4.4. (i) If $(\bar{q}, u) \in \mathscr{E}_{R}$ and $f(u)=0$, then the linearization $D(\pi \oplus f)(\bar{q}, u)$ is a semi-Fredholm operator.

(ii) $D(\pi \oplus f)\left(q_{0}, x_{0}\right)$ is an isomorphism.

(iii) $(\pi \oplus f)^{-1}\left(q_{0}, 0\right)=\left\{\left(q_{0}, x_{0}\right)\right\}$.

Now we come to the main result of this section. For a nonlinear $C^{2}$ Fredholm operator $F: X \rightarrow Y$ of index 0 between Banach manifolds, $\mathscr{U} \subset X, F \mid \overline{\text { proper, }}$ and $y \in Y \backslash F(\partial \mathscr{U})$, let $\operatorname{deg}(F, \mathscr{U}, y)$ be the $Z_{2}$ degree as defined in [Sm]. Let $\Omega_{T}^{\text {contr }}$ be the elements of $\Omega_{T}$ which are contractible.

Proposition 4.5. For each compact $K \subset \Omega_{T}^{\text {contr }}$, there exists an open $\mathscr{Q} \subset \boldsymbol{\delta}_{R}$ such that:

(i) $\left.\pi \oplus f\right|_{\bar{x}}$ is a proper Fredholm operator of index 0 .

(ii) $\operatorname{deg}(\pi \oplus f, \mathscr{U},(\bar{q}, 0))=1$ for all $\bar{q} \in K$.

Proof. Enlarging $K$ if necessary, we may assume that it is connected and contains $q_{0}$. By Proposition 3.7, $(\pi \oplus f)^{-1}(K \times\{0\})$ is compact. Since the semiFredholm operators form an open set, by 4.4 (i) we find a neighborhood $\mathscr{U}_{1}$ of $(\pi \oplus f)^{-1}(K \times\{0\})$ on which $\pi \oplus f$ is semi-Fredholm. Taking the connected component $\mathscr{U}_{2}$ of $(\pi \oplus f)^{-1}\left(\left(q_{0}, 0\right)\right)$ in $\mathscr{U}_{1}$, Corollary 4.4 (ii) and the fact that the set of Fredholm operators of index 0 is open and closed in the set of semi-Fredholm operators yield that $\left.\pi \oplus f\right|_{\boldsymbol{w}_{2}}$ is Fredholm of index 0 . Fredholm operators are locally proper ([Sm], Theorem 1.6), so we find a neighborhood $\mathscr{U}$ of $(\pi \oplus f)^{-1}(K \times\{0\})$ $\cap \mathscr{U}_{2}$ in $\mathscr{U}_{2}$ such that $\left.\pi \oplus f\right|_{\mathbf{R}}$ is proper.

Because $K$ is connected, $K \times\{0\}$ is contained in one connected component of $\Omega_{T} \times F$. By definition of the $Z_{2}$ degree, $\operatorname{deg}(\pi \oplus f, \mathscr{Q},(\bar{q}, 0))$ is the same for all $\bar{q} \in K$. But from 4.4 (ii), (iii) we know that $\left(q_{0}, 0\right)$ is a regular value of $\left.\pi \oplus f\right|_{*}$ whose preimage consists of one single point. Thus $\operatorname{deg}\left(\pi \oplus f, \mathscr{q l}\left(q_{0}, 0\right)\right)=1$, which concludes the proof.

This is the desired existence result for finite flow lines. However, in order to pass to infinite flow lines we want to interpret this result in terms of cohomology. Let $\bar{H}^{*}$ denote the Alexander-Spanier cohomology as defined in [Sp].

LemmA 4.6. For each nontrivial cohomology class $0 \neq \alpha \in \bar{H}^{*}\left(\Omega_{T}^{\text {contt }}, \mathbf{Z}_{2}\right)$, there exists a compact $K \subset \Omega_{T}^{\text {contr }}$ such that, for all $R>0$,

$$
\left(\left.\pi\right|_{A_{k}}\right)^{*} \alpha \neq 0,
$$

where $A_{R}=(\pi \oplus f)^{-1}(K \times\{0\}) \subset \mathscr{E}_{R}$.

Proof. [Ci], Proposition 6.3. For the convenience of the reader we will recall the basic ideas. First one constructs a connected compact finite-dimensional $C^{1}$. manifold $K$ with boundary and an embedding $i: K \hookrightarrow \Omega_{T}^{\text {contr }}$ such that $i^{*} \alpha \neq 0$. By an approximation argument we may assume the embedding $i \oplus 0: K \hookrightarrow \Omega_{T}^{\text {conir }} \times$ $F$ to be transversal to $\left.\pi \oplus f\right|_{\overline{\mathbb{Z}}}$, with $\mathscr{Q}$ from Proposition 4.5. 
Then $A=(\pi \oplus f \mid \bar{z})^{-1}(K \times\{0\})$ is a compact finite-dimensional manifold with boundary, and $\left.\pi\right|_{A}: A \rightarrow K$ is a mapping of $\mathbf{Z}_{2}$-degree 1 . It is a simple consequence of Poincaré-Lefschetz duality that under these circumstances $\left(\left.\pi\right|_{A}\right)^{*}: \bar{H}^{*}(K)$ $\rightarrow \bar{H}^{*}(A)$ is injective in all dimensions. In particular $\left(\left.\pi\right|_{A}\right)^{*} \alpha \neq 0$, and because $A \subset A_{R}$, also $\left(\left.\pi\right|_{A_{R}}\right) * \alpha \neq 0$.

Now we are in a position to pass to the limit $R \rightarrow \infty$ and show that the existence result in the form of Lemma 4.6 survives in this process. Let $X$ be defined as in Section 3 and

$$
\Omega:=\left\{q \in C^{0}(\mathbb{R}, M) \mid \lim _{t \rightarrow \pm \infty} q(t)=q_{0}\right\},
$$

equipped with the topology induced by the norm

$$
\sup _{t \in \mathbf{R}}|q(t)|
$$

We have a continuous projection $\pi: X \rightarrow \Omega$ given by

$$
\pi u(t):=\tau u(0, t)
$$

where $\tau: T M \rightarrow M$ is the projection. As usual, by $X^{\text {contr }}$ and $\Omega^{\text {contr }}$ we denote the subsets of contractible elements of these spaces.

The result we want to prove is the following.

Proposition 4.7. If $\mathscr{C} / \mathbf{Z}$ is finite, $\pi: X^{\text {contr }} \rightarrow \Omega^{\text {contr }}$ induces an injection

$$
\pi^{*}: \bar{H}^{*}\left(\Omega^{\text {contr }}, \mathbf{Z}_{2}\right) \rightarrow \bar{H}^{*}\left(X^{\text {contr }}, \mathbf{Z}_{2}\right)
$$

Proof. First we notice that the inclusion $i: \Omega_{T} \hookrightarrow \Omega$ is a homotopy equivalence. This follows from the fact that the homeomorphism given in [Gi], Lemma 3.7, is homotopic to the inclusion.

This allows us to replace $\alpha$ and $\pi$ in Lemma 4.6 by an $\alpha \in \bar{H}^{*}\left(\Omega^{\text {contr }}, Z_{2}\right)$ and the projection $\pi: \mathscr{E}_{R} \rightarrow \Omega$. Next, we observe that because $\mathscr{J}: \Omega_{T} \rightarrow \Omega$ is homotopic to the inclusion, $\left.\pi\right|_{A_{2 R}}$ is homotopic to the projection $\pi_{R}: A_{2 R} \rightarrow \Omega$ given by $\pi_{R} u(t)$ $:=\tau u(R, t)$. Thus we can reformulate Lemma 4.6 as follows: For each $0 \neq \alpha \in$ $\bar{H}^{*}\left(\Omega^{\text {contr }}, \mathbf{Z}_{2}\right)$ there exists a compact $K \subset \Omega_{T}^{\text {contr }}$ such that for all $R>0$,

$$
0 \neq \pi_{R}^{*} \alpha \in \bar{H}^{*}\left(A_{2 R}, \mathbf{Z}_{2}\right)
$$

Now we fix such an $\alpha$ and $K$. For each $R>0$ we choose mappings $\phi_{R} \in C^{\infty}(\mathbf{R}$, $[0,2 R])$ with $\phi_{R}(s)=s+R$ for $|s| \leqslant R-1, \phi_{R}(s) \equiv 0$ for $s \leqslant-R, \phi_{R}(s) \equiv 2 R$ for 
$s \geqslant R$, and we define

$$
\begin{aligned}
P: A_{2 R} & \rightarrow \mathscr{E} \\
(\bar{q}, u) & \mapsto P u(s, t):=u\left(\phi_{R}(s), t\right),
\end{aligned}
$$

where $\mathscr{E}$ is defined at the beginning of Section 3 .

We claim that for every neighborhood $\mathscr{U}$ of $X^{\text {contr }}$ in $\mathscr{E}$ we can find an $R>0$ such that $P\left(A_{2 R}\right) \subset \mathscr{U}$. Otherwise there would exist sequences $R_{n} \rightarrow \infty$ and $\left(\bar{q}_{n}, u_{n}\right)$ $\in A_{2 R_{n}}$ with $P u_{n} \notin \mathscr{U}$.

Since the $u_{n}$ satisfy boundary conditions with the same compact $f(K) \subset \Omega_{T}^{\infty}$, from Proposition 3.9 and the Arzela-Ascoli theorem, $\left(P u_{n}\right)$ has a subsequence converging in the topology of $\mathscr{E}$. Moreover, we have an upper estimate on $\left\|\partial_{s} P u_{n}\right\|_{L^{2}\left(R^{2}\right)}$ independent of $n$, and for $s \in\left[-R_{n}+1, R_{n}-1\right]$, we have

$$
\bar{\partial} P u_{n}(s, t)+H^{\prime}\left(t, P u_{n}(s, t)\right)=0 .
$$

So the subsequence of $\left(P u_{n}\right)$ converges in the topology of $\mathscr{E}$ to some $u \in X$, which contradicts the assumption $P u_{n} \notin \mathscr{U}$.

From $\pi_{R}^{*} \alpha \neq 0, P\left(A_{2 R}\right) \subset \mathscr{Q}$, and $\pi \circ P=\pi_{R}$, it follows that $\left.\pi\right|^{*} \alpha \neq 0$. Since this is true for all neighborhoods $\mathscr{U}$ of $X$ in $\mathscr{E}$, the tautness of Alexander-Spanier cohomology ([Sp], Theorem 6.2) implies that $\left(\left.\pi\right|_{X_{\text {conors }}}\right)^{*} \alpha \neq 0$.

5. Proof of Theorem 2.1 in the case " $\pi_{1}(M)$ finite". We are going to show that if $\pi_{1}(M)$ is finite, then the conclusions of Corollary 3.10 and Proposition 4.7 lead to a contradiction, thus proving that the assumption $\mathscr{C} / \mathrm{Z}$ finite must be wrong. This will be due to the following result by D. Sullivan [Su].

Proposition 5.1. If $\pi_{1}(M)$ is finite, then $\bar{H}^{k}\left(\Omega, Z_{2}\right) \neq\{0\}$ for infinitely many $k \in \mathbb{N}$.

Proof. If $\pi_{1}(M)$ is finite, the universal covering $\tilde{M}$ is a compact manifold. In [Su], page 46, it is shown that infinitely many rational Betti numbers of the loop space $C^{0}\left(S^{1}, \tilde{M}\right)$ are nonzero. Because of the fibring of $C^{0}\left(S^{1}, \tilde{M}\right) \rightarrow \tilde{M}$ with fibre $C^{0}\left(S^{1}, \tilde{M}, \tilde{q}_{0}\right)=\left\{x \in C^{0}\left(S^{1}, \tilde{M}\right) \mid x(0)=\tilde{q}_{0}\right\}$, the same is true for $C^{0}\left(S^{1}, \tilde{M}, \tilde{q}_{0}\right)=$ $C_{\text {contr }}^{0}\left(S^{1}, M, q_{0}\right)$. Since $C_{\text {contr }}^{0}\left(S^{1}, M, x_{0}\right)$ is a Banach manifold, Alexander-Spanier cohomology and simplicial cohomology agree on it, and we conclude that $\bar{H}^{k}\left(C_{\text {contr }}^{0}\left(S^{1}, M, q_{0}\right), Z_{2}\right) \neq\{0\}$ for infinitely many $k \in \mathbb{N}$. The homotopy equivalence between $\Omega$ and $C_{\text {contr }}^{0}\left(S^{1}, M, q_{0}\right)$ ([Gi], Lemma 3.7) now gives the result.

On the other hand, Corollary 3.10 implies the following.

Proposition 5.2. If $\mathscr{C} / \mathbf{Z}$ is finite, then there exists a $k_{0} \in \mathbb{N}$ such that

$$
\pi^{*}: H^{k}(\Omega, G) \rightarrow H^{k}(X, G)
$$

is the zero map for every $k \geqslant k_{0}$ and each group $G$. 
Proof. Let $*$ be the action of $\mathrm{Z}$ on $\mathscr{E}$ defined by $n * u(s, t)=u(s, t-n)$, and let $p: \mathscr{E} \rightarrow \mathscr{E} / \mathbf{Z}$ be the canonical projection. There exists a covering of $X / \mathbf{Z} \subset \mathscr{E} / \mathbf{Z}$ by a family $\left(V_{\alpha}\right)_{\alpha \in A}$ of open subsets of $\mathscr{E} / \mathbf{Z}$, such that $p^{-1}\left(V_{\alpha}\right)=\left\{n * W_{\alpha} ; n \in \mathbf{Z}\right\}$ for any $\alpha \in A$, where the sets $W_{\alpha}$ are open in $\mathscr{E}$ and satisfy:

(a) For some $\alpha_{0}, W_{\alpha_{0}}$ is a neighborhood of the constant curve $x_{0}$ in $\mathscr{E}$ and it is invariant by $\mathbf{Z}$.

(b) If $\alpha \neq \alpha_{0}$ and $n \neq 0$, then $W_{\alpha} \cap n * W_{\alpha}=\varnothing$.

(c) Any finite intersection of open sets of the form $n * W_{a}$ is contractible.

To construct $\left(V_{\alpha}\right)_{\alpha \in A}$, we consider, for $b$ large enough, an isometric embedding of $M$ in $\mathbf{R}^{b}$, which allows us to identify $\mathscr{E}$ with a Hilbert submanifold of the Hilbert space $H=W_{\text {semiloc }}^{2,2}\left(\mathbb{R}^{2}, \mathbb{R}^{2 b}\right)$. Our set $A$ is $X / Z$ itself. We take $\alpha_{0}=p\left(x_{0}\right)$, and for any $\alpha \in A, W_{\alpha}=B_{\alpha} \cap \mathscr{E}$, where $B_{\alpha}$ is a ball in $H$ centered at some point of $p^{-1}(\alpha)$, with small radius. Property (a) is clearly true, and properties (b) and (c) are satisfied if the radii of the balls $B_{\alpha}$ are chosen small enough.

Now, by Corollary 3.10, we can extract from $\left(V_{\alpha}\right)_{\alpha \in A}$ a finite subcovering $\left(V_{a(i)}\right)_{0 \leqslant i \leqslant k_{0}-1}$. Properties (a) and (b) imply that any intersection of $k_{0}+1$ distinct sets of the form $n * W_{a(i)}$ is empty.

So, denoting

$$
\mathscr{W}=\bigcup_{\substack{0 \leqslant i<k_{0} \\ n \in \mathbf{Z}}} n * W_{\alpha(i)},
$$

from (c) the $k_{0}$ th Cech homology group of $\mathbb{W}$ is zero. But $\mathscr{W}$ is a metric space, so the Cech and Alexander-Spanier cohomologies on $\mathbb{W}$ are the same (see [Sp]).

Denoting by $j$ the canonical injection of $X$ in $\mathscr{W}$ and by $\bar{\pi}$ the natural extension of $\pi$ to $\mathbb{W}$, the proposition then follows from the formula $\pi^{*}=j^{*} \circ \bar{\pi}^{*}$.

The conclusions of Propositions 4.7, 5.1 and 5.2 obviously contradict one another. Thus the assumption $\mathscr{C} / \mathbf{Z}$ finite must be wrong, which proves Theorem 1.1 in the case " $\pi_{1}(M)$ finite".

6. Proofs of Theorem 1.1 (case " $\pi_{1}(M)$ infinite") and Theorem 1.2. To prove Theorem 1.1 (case " $\pi_{1}(M)$ infinite") and Theorem 1.2, we have to study noncontractible pseudoholomorphic curves.

We take a smooth curve $\bar{q} \in \Omega_{T}^{\infty}$, which represents a nontrivial class in $\pi_{1}(M)$. We are going to prove the following result, which is the analogue of Proposition 4.5 in the noncontractible case.

Proposition 6.1. Assume (H1)-(H4) are satisfied. Then $X_{\bar{q}, \mathbb{R}}$ is nonempty for any $R>0$.

Proof. We cannot prove Proposition 6.1 by a homotopy between $\bar{q}$ and $q_{0}$ as in the case of Proposition 4.5. Instead of this argument, we will use a homotopy between Hamiltonians.

We define a 1-parameter family of Hamiltonians on $T M$,

$$
H_{\lambda}(t, x)=\lambda H_{1}(t, x)+(1-\lambda) H_{0}(t, x),
$$


where $H_{0}(t, x)=H(t, x)$ is the Hamiltonian of Theorems 1.1, 1.2, and

$$
H_{1}(t, x)=\theta(t)\left(\alpha_{i}(p)+\frac{|p|^{2}}{2}\right)+(1-\theta(t)) H(t, x)
$$

Here,

$$
\begin{gathered}
\theta \in C^{\infty}(\mathbf{R},[0,1]), \\
\theta \equiv 1 \text { on }[-T, T], \\
\theta \equiv 0 \text { on } \mathbb{R} \backslash[-2 T, 2 T] .
\end{gathered}
$$

For any $t \in \mathbb{R}, \alpha_{t}$ is a 1 -form on $M$ such that

$$
\left\langle\alpha_{t}(\bar{q}(t)), p\right\rangle=\langle\dot{\bar{q}}(t), p\rangle, \quad \forall p \in T_{\bar{q}(t)} M
$$

We introduce

$$
X_{\bar{q}, R}^{\lambda}=\left\{u \in H^{2,2}\left(Z_{R}\right) \mid \bar{\partial} u+H_{\lambda}^{\prime}(t, u)=0 \text { and } u(0, \cdot) \in M, \tau u(R, \cdot)=\bar{q}\right\} .
$$

LeMmA 6.2. $R, \bar{q}$ being fixed, there is a constant $C>0$ such that $\|u\|_{H^{1.2}} \leqslant C$, for any $\lambda \in[0,1]$ and $u \in X_{\bar{q}, R}^{\lambda}$.

Proof. As in the discussion before Lemma 3.3, one shows

$$
\begin{aligned}
& I(u(R)) \leqslant c\left(\|\dot{\bar{q}}\|_{c_{0}}\right), \\
& I(u(0)) \geqslant 0 .
\end{aligned}
$$

This implies

$$
\int\left|\partial_{s} u\right|^{2} d s d t \leqslant c\left(\|\dot{\bar{q}}\|_{c^{\circ}}\right)
$$

Denoting by $(q, p)=u$ the base and fibre parts of $u$, we obtain

$$
\begin{aligned}
\|q(s)\|_{L^{2}(d t)} & \leqslant\|q(R)\|_{L^{2}(d t)}+\int_{s}^{R}\left\|\partial_{s} q\right\|_{L^{2}(d t)} d s \\
& \leqslant\|\bar{q}\|_{L^{2}}+\sqrt{R}\left\|\partial_{s} q\right\|_{L^{2}(d s a t)} \\
& \leqslant c(\bar{q}, R) \text { for all } s \in[0, R] .
\end{aligned}
$$


If $|p|$ is the norm in the fibre and $\nabla_{s}$ the covariant derivative with respect to the Levi-Civita connection, we have

$$
\partial_{s}|p|=\frac{1}{2|p|} \partial_{s}|p|^{2}=\frac{\left\langle p, \nabla_{s} p\right\rangle}{|p|}
$$

and thus

$$
\begin{aligned}
\|p(s)\|_{L^{2}(d t)} & \leqslant\|p(0)\|_{L^{2}(d t)}+\int_{0}^{s}\left\|\nabla_{s} p\right\|_{L^{2}(d t)} d s \\
& \leqslant \sqrt{R}\left\|\nabla_{s} p\right\|_{L^{2}(d s d t)} \\
& \leqslant c(\bar{q}, R) \text { for all } s \in[0, R] .
\end{aligned}
$$

Hence $\|u\|_{L^{2}(d s d t)} \leqslant c(\bar{q}, R)$, and using the differential equation yields the result.

LEMMA 6.3. $\bar{q}$ being fixed, there is an $R_{0}>0$ such that, for any $\alpha \in \mathbb{N}^{2},\left|\partial^{\alpha} u(s, t)\right|$ $\leqslant C_{\alpha}$, where $C_{\alpha}$ is independent of $(s, t) \in Z_{R_{0}}$ and $u \in X_{\bar{q}, R_{0}}^{\lambda}, \lambda \in(0,1)$.

Proof. The estimate $|u(s, t)| \leqslant C_{(0,0)}$ is obtained as in the proof of Lemma 3.3. The only difference is that $H_{2}$ does not necessarily satisfy assumption (H3), so that Lemma 2.2 cannot be used. So we cannot cut $Z_{R}$ into "narrow strips". To overcome this problem, we choose $R_{0}$ small enough so that $Z_{R_{0}}$ is itself a "narrow strip".

The estimates for $\alpha \neq(0,0)$ are obtained exactly as in the proof of Lemma 3.4.

Lemma 6.4. $\bar{q}$ is fixed, and $R_{0}$ is chosen as in Lemma 6.3. Then, for any $\alpha \in \mathbb{N}^{2}$, there are $K_{\alpha}, \rho_{\alpha}>0$ such that

$$
\left|\partial^{\alpha} u(s, t)\right| \leqslant C_{\alpha} e^{-\rho_{\alpha}|t|},
$$

for any $(s, t) \in Z_{R_{0}}$ and $u \in X_{\bar{q}, R_{0}}^{\lambda}, \lambda \in[0,1]$.

Proof. The proof of Lemma 6.4 is identical to that of Lemma 3.6.

As in Section 4, we now define the mapping

$$
f_{\lambda}(u)=\pi_{F} \circ \psi\left(\bar{\partial} u+H_{\lambda}^{\prime}(t, u)\right),
$$

and we have another lemma.

LEMMA 6.5. If $u \in H^{2,2}\left(Z_{R_{0}}, T M\right)$ and $f_{\lambda}(u)=0$, then $D f_{\lambda}(u)$ is a semi-Fredholm operator. 
The proof is similar to that of Lemma 4.3.

LEMMA 6.6. The only solution to the equation $f_{1}(u)=0$ in $\mathscr{E}_{\bar{q}, R_{0}}$ is $\bar{u}(s, t)=$ $(\bar{q}(t), 0)$, and $D f_{1}(\bar{u})$ is an isomorphism.

Proof. Let $I_{1}(x):=\int_{R} p d q-H_{1}(t, x)$. (i) Any $u \in \mathscr{E}_{\bar{q}, R_{0}}$ must satisfy $\left.I_{1}\left(u, R_{0}\right)\right) \leqslant$ 0 and $I_{1}(u(0)) \geqslant 0$, by construction of $H_{1}$. So, if $f_{1}(u) \stackrel{q}{=} 0$, then $\int_{z_{R_{0}}}\left|\partial_{s} u\right|^{2}=0$, and hence $u=\bar{u}$.

(ii) $\bar{x}(t)=(\bar{q}(t), 0)$ is a critical point of the functional $I_{1}$ in $H^{1,2}(R, T M)$. Its Hessian is $\bar{I}(y)=I_{1}^{\prime \prime}(\bar{x}) \cdot(y, y)$, and we have

$$
I_{1}\left(\exp _{\bar{x}} y\right)=I_{1}(\bar{x})+\frac{1}{2} I_{1}^{\prime \prime}(\bar{x}) \cdot(y, y)+o\left(\|y\|_{H^{1,2}}^{2}\right) .
$$

Moreover, $D f_{1}(\bar{u}) \cdot y=\nabla_{s} y-\nabla \bar{I}(y)$, where $\nabla \bar{I}$ is the $L^{2}$-gradient of $\bar{I}$.

We always have, for $v \in T_{\bar{u}} \mathscr{E}_{\bar{q}, R_{0}}$,

$$
\begin{aligned}
0 & \geqslant I_{1}\left(\exp _{\bar{u}} v(R, \cdot)\right)-I_{1}\left(\exp _{\bar{u}} v(0, \cdot)\right) \\
& =\frac{1}{2}(\bar{I}(v(R, \cdot))-\bar{I}(v(0, \cdot)))+o\left(\|v(R, \cdot)\|_{H^{1,2}}^{2}+\|v(0, \cdot)\|_{H^{1.2}}^{2}\right) .
\end{aligned}
$$

Since $\bar{I}$ is a quadratic form this implies, for any $v \in T_{\bar{u}} \mathbb{E}_{\bar{q}, R_{0}}$,

$$
\begin{aligned}
0 & \geqslant \bar{I}(v, R, \cdot))-\bar{I}(v(0, \cdot)) \\
& =\int_{0}^{R_{0}}\left\langle\nabla_{s} v(s), \nabla \bar{I}(v(s))\right\rangle_{L^{2}(\mathbf{R})} d s \\
& \geqslant\|\nabla \bar{I}(v)\|_{L^{2}\left(Z_{Z_{0}}\right)}^{2}-\left\|D f_{1}(\bar{u}) v\right\|_{L^{2}\left(Z_{R_{0}}\right)}\|\nabla \bar{I}(v)\|_{L^{2}} .
\end{aligned}
$$

Hence

$$
\|\nabla \bar{I}(v)\|_{L^{2}} \leqslant\left\|D f_{1}(\bar{u}) v\right\|_{L^{2}},
$$

and similarly

$$
\left\|\nabla_{s} v\right\|_{L^{2}} \leqslant\left\|D f_{1}(\bar{u}) v\right\|_{L^{2}} .
$$

As in the proof of Lemma 6.2, we deduce $\|v\|_{H^{1,2}} \leqslant c\left\|D f_{1}(\bar{u}) v\right\|_{L^{2}}$. By the same argument applied to the derivatives $\nabla_{s} v$ and $\nabla_{t} v$, we finally get

$$
\|v\|_{H^{2,2}} \leqslant c\|D f(\bar{u}) v\|_{H^{1,2}} .
$$

To prove the surjectivity of $D f_{1}(\bar{u})$, we trivialize the bundle $\bar{q}^{*} T T M \rightarrow \mathbb{R}$ such that the trivialization is constant over $(-\infty,-2 T] \cup[2 T, \infty)$, and multiplication 
by $J(\bar{q}(t))$ corresponds to multiplication by $i$ in $\mathbb{C}^{n}$. This is possible because the structure group $U(n)$ is connected. However, this trivialization need not respect the splitting

$$
T T M=T^{h} T M \oplus T^{v} T M
$$

In fact, the following two situations may occur: either under the trivialization $T^{h} T M \triangleq \mathbb{R}^{n}$ and $T^{v} T M \triangleq i \mathbb{R}^{n}$, or $T_{\bar{q}(t)}^{h} T M \triangleq\left(e^{i \pi \varphi(t)} \mathbb{R}\right) \oplus \mathbb{R}^{n-1}$ and $T_{\bar{q}(t)}^{v} T M \hat{=}$ $\left(e^{i \pi \varphi(t)} i \mathbb{R}\right) \oplus i \mathbb{R}^{n-1}$, where $\varphi: \mathbb{R} \rightarrow[0,1]$ is $\equiv 0$ for $t \leqslant-T$ and $\equiv 1$ for $t \geqslant T$. Choose a natural number $N>2 T$, extend $\left.\bar{q}\right|_{[-N, N]}$ to a $2 N$-periodic loop $\bar{q}_{N}$, and regard $D f_{1}(\bar{u})$ as an operator

$$
g_{N}: E_{N} \rightarrow F_{N},
$$

where

$$
\begin{gathered}
E_{N}=\left\{\eta \in H^{2,2}\left([0, R] \times \mathbb{R} / 2 n Z, \bar{u}_{N}^{*} T T M\right) \mid \eta(0, t) \in T_{\bar{q}(t)}^{h} T M, \eta(R, t) \in T_{\bar{q}(t)}^{v} T M\right\}, \\
F_{N}=H^{1,2}\left([0, R] \times \mathbb{R} / 2 N Z, \bar{u}_{N}^{*} T T M\right) .
\end{gathered}
$$

As above, one shows that $g_{N}$ is injective. Moreover, under trivialization, $g_{N}$ corresponds to an operator $\partial_{s}+i \partial_{t}+A(t)$ in $\mathbb{C}^{n}$, and from the discussion above, the loops of Lagrangian subspaces $T_{\bar{q}(t)}^{h} T M$ and $T_{\bar{q}(t)}^{v} T M$ have the same Maslov index. So by [Ci], Theorem 2.3, the Fredholm index of $g_{N}$ is 0 . This implies that $g_{N}$ is surjective. As in the proof of Lemma 4.2, an approximation argument ends the proof of Lemma 6.6 .

As a consequence of Lemmas 6.5 and 6.6, there exists an open subset $\mathscr{Q}$ of $\mathscr{G}_{\bar{q}, R_{0}}$ such that $\left.f_{1}\right|_{x}$ is Fredholm of index 0 and $\operatorname{deg}_{\mathbf{z}_{2}}\left(f_{1}, \mathscr{U}, 0\right)=1$. From Lemmas 6.4 and 6.5, this is also the case for $f_{0}$ on some $\mathscr{U}_{R_{0}} \subset \mathscr{E}_{q, R_{0}}$. By Proposition 3.7 and Lemma 6.5 , for any $R \geqslant 0$, there exists an open set $\mathscr{U}_{R} \subset \mathscr{E}_{\bar{q}, R}$ such that $f_{0} l_{\boldsymbol{x}_{R}}$ is Fredholm of index 0 and $\operatorname{deg}_{\mathbf{z}_{2}}\left(f_{0}, \mathscr{U}_{R}, 0\right)=1$. This proves Proposition 6.1.

We now prove Theorem 1.1 (case " $\pi_{1}(M)$ infinite"). Assume by contradiction that $\mathscr{C} / \mathbf{Z}$ is finite (i.e., $(\mathscr{H})$ is satisfied). Take a sequence $\boldsymbol{R}_{n} \rightarrow+\infty$, and $u_{n} \in X_{\bar{q}, R_{n}}$. Then, from Proposition 3.9, $u_{n}$ has a limit $u$ for the $H_{\text {semiloc }}^{2,2}$ topology.

For each $s \in \mathbb{R}, \tau u(s, \cdot)$ represents the same class as $\bar{q}$ in $\pi_{1}(M)$. So the limit $u_{+}$of $u(s, \cdot)$ as $s \rightarrow+\infty$ given by Lemma 3.2 is a homoclinic orbit such that $\tau u_{+}$is in the class of $\bar{q}$. Taking infinitely many classes, we get a contradiction, and Theorem 1.1 is proved in the case " $\pi_{1}(M)$ infinite".

We end this paper with the proof of Theorem 1.2. Once again, we take $R_{n} \rightarrow \infty$ and $u_{n} \in X_{\bar{q}, R_{n}}$. Since $\int_{Z_{R_{n}}}\left|\partial_{s} u_{n}\right|^{2} d s d t$ is bounded independently of $n$, we find a sequence $s_{n} \in\left[0, R_{n}\right]$ such that $I^{\prime}\left(u\left(s_{n}\right)\right) \rightarrow 0$ in $L^{2}, I\left(u\left(s_{n}\right)\right)$ bounded. Then, from Proposition 2.5, there is a subsequence $x_{n}$ of $u_{n}\left(s_{n}, \cdot\right)$ such that

$$
\left\|x_{n}-\bar{p}_{n} * \bar{x}\right\|_{H^{1,2}} \rightarrow 0 \text { as } n \rightarrow \infty,
$$


where $m \in \mathbb{N}, \bar{x}=\left(x^{1}, \ldots, x^{m}\right) \in \mathscr{C}^{m}, \overline{p_{n}}=\left(p_{n}^{1}, \ldots, p_{n}^{m}\right) \in \mathbf{Z}^{m}$, and

$$
\left(p_{n}^{i+1}-p_{n}^{i}\right) \underset{n \rightarrow \infty}{\longrightarrow}+\infty \quad(\forall i) \text {. }
$$

But in $\pi_{1}(M)$,

$$
[\bar{q}]=\left[\tau x_{n}\right]=\left[\tau x^{m}\right] \cdot\left[\tau x^{m-1}\right] \cdot \cdots \cdot\left[\tau x^{1}\right],
$$

and Theorem 1.2 is proved by choosing arbitrary classes $[\bar{q}]$.

\section{REFERENCES}

[A] N. ARonszann, A unique continuation theorem for solutions of elliptic partial differential equations or inequalities of second order, J. Math. Pures Appl. (9) 36 (1957), 235249.

[Am] H. AMANN, Fixed point equations and nonlinear eigenvalue problems in ordered Banach spaces, Siam Rev. 18 (1975), 620-709.

[B] S. V. Bolotin, Libration motions of natural dynamical systems, Vestnik Moskov. Univ. Ser. I Mat. Mekh. 6 (1978), 72-77.

[BGi] V. BENCI AND F. GianNon, Homoclinic orbits on compact manifolds, J. Math. Anal. Appl. 157 (1991), 568-576.

[Ci] K. Cielebax, Pseudo-holomorphic curves and periodic orbits on cotangent bundles, J. Math. Pures Appl. 73 (1994), 251-278.

[CZES] V. CotI ZeLATI, I. EKELAND, AND E. SERE, A variational approach to homoclinic orbits in Hamiltonian systems, Math. Ann. 288 (1990), 133-160.

[CZR] V. COTI ZELATI AND P. RABINOWTIZ, Homoclinic orbits for second-order Hamiltonian systems possessing superquadratic potentials, preprint, 1991.

[El] H. I. Eliasson, Geometry of manifolds of maps, J. Differential Geom. 1 (1967), 169-194.

[Fe] P. FELMFR, Heteroclinic orbits for spatially periodic Hamiltonian systems, Ann. Inst. H. Poincaré Anal. Non Linéaire 8 (1991), 477-497.

[F1] A. FlOER, The unregularized gradient flow of the symplectic action, Comm. Pure Appl. Math. 41 (1989), 775-813.

[Gi] F. GIANNON, On the existence of homoclinic orbits on Riemannian manifolds, preprint, Univ. of Wisconsin-Madison, 1991.

[Gr] M. Gromov, Pseudo holomorphic curves in symplectic manifolds, Invent. Math. 82 (1985), 307-347.

[GiR] F. GIANNONI AND P. RABINOWTtz, Multibump homoclinic solutions for Lagrangian systems on Riemannian manifolds, preprint, 1992.

[H] H. HoFER, Liusternik-Schnirelman theory for Lagrangian intersections, Ann. Inst. H. Poincaré Anal. Non Linéaire 5 (1988), 465-499.

[HW] H. HOFER AND K. WYSOCKI, First order elliptic systems and the existence of homoclinic orbits in Hamiltonian systems, Math. Ann. 288 (1990), 483-503.

[Li] Y. Y. Ll, On $-\Delta u=k(x) u^{5}$ in $\mathbf{R}^{3}$, preprint, Rutgers Univ.

[L] P. L. Lions, The concentration-compactness principle in the Calculus of Variations, Rev. Mat. Iberoamericana 1 (1985), 145-201.

[Pa] R. S. Palais, Foundations of Global Nonlinear Analysis, Benjamin, New York, 1968.

[R] P. RABINowItZ, Periodic and heteroclinic orbits for a periodic Hamiltonian system, Ann. Inst. H. Poincaré Anal. Non Linéaire 6 (1989), 331-346.

[S1] E. SERE, Existence of infinitely many homoclinic orbits in Hamiltonian systems, Math. Z. 209 (1992), 27-42. 
[S2] - Looking for the Bernoulli shift, preprint, CEREMADE, 1992.

[Sm] S. SMALE, An infinite dimensional version of Sard's Theorem, Amer. J. Math. 87 (1965), 861-866.

[Sp] E. H. SPANIER, Algebraic Topology, McGraw-Hill, New York, 1966.

[St] M. STRUWE, A global compactness result for elliptic boundary value problems involving limiting nonlinearities, Math. Z. 187 (1984), 511-517.

[Su] D. Sullivan, Differential forms and the topology of manifolds, Proc. Int. Conf. on Manifolds and Related Topics in Topology, Tokyo (1973), 37-49.

Cieliebak: Mathematik, ETH-Zentrum, CH-8092 ZÜrich, Switzerland; cielieba@math.ethz.ch SÉre: CEREMADE, Universitt Paris-Dauphine, Place de Lattre de Tassigny, 75775 Paris cedex 16, FrancE; sere@paris9.dauphine.fr 